\title{
Static properties of two linearly coupled discrete circuits
}

\author{
Albert Escrivà,, , 2, f Andrea Richaud, ${ }^{3}$ Bruno Juliá-Díaz, ${ }^{1,2}$ and Montserrat Guilleumas ${ }^{1,2}$ \\ ${ }^{1}$ Departament de Física Quàntica i Astrofísica, Facultat de Física, \\ Universitat de Barcelona, Martí $i$ Franquès 1, 08028 Barcelona, Spain \\ ${ }^{2}$ Institut de Ciències del Cosmos, Universitat de Barcelona, 08028 Barcelona, Spain \\ ${ }^{3}$ Scuola Internazionale Superiore di Studi Avanzati (SISSA), Via Bonomea 265, I-34136, Trieste, Italy
}

\begin{abstract}
Bosonic two-ring ladders constitute an important class of atomtronic circuits, where coherent current flows not only can offer a new insight into many-body physics, but also can play the role of actual degrees of freedom, and hence allow for a viable implementation of cold-atom based devices and qubit systems. In this work, we exhaustively investigate the ground state properties and the lowlying energy spectrum of two linearly coupled Bose-Hubbard rings. We show that the competition among interactions, intra- and inter-ring hopping processes gives place to a rather rich physical scenario, where Mott-like states and (different kinds of) superfluid-like states emerge. The latter ones depend also on the (in)commensurate filling of the atoms. Our analysis, carried out within a simple analytical framework and by means of the exact numerical diagonalization of the system Hamiltonian, provides one with a rather complete characterization of the static properties of the tworing ladder, including, but not limited to, coherence, fragmentation, correlations, and entanglement. We complement our investigation by studying how these indicators depend on the commensurability of the total number of bosons with respect to the total number of sites and show that the two stacked rings are always entangled for an odd number of atoms.
\end{abstract}

\section{INTRODUCTION}

Ultracold quantum gases in closed geometries have become a basic building block of quantum technologies. The versatility and high degree of experimental control over the interactions and the geometry of ultracold atoms have made it possible to engineer atomic devices with different types of geometries and couplings, in particular, rings and ladders. This experimental progress has boosted the appearance of the emergent field of Atomtronics in quantum technologies [1 5]. The aim is to design atomtronic circuits by coupling simple elements capable of producing complex applications with matter waves. For instance, quantum transport through different parts of the atomtronic circuit [6], the emulation of superconducting flux qubits used in quantum computing [7], or production of entangled states. Entanglement is an important feature of quantum systems, that can be used for quantum information processing. Atomtronic devices may then be used to simulate intricate quantum systems 8, to develop finer sensors and improve our metrological capabilities [1, 9, 10].

The characterization of ground state properties, correlations, entanglement and energy spectrum of ultracold bosons in a ring lattice with few sites has been extensively investigated in the literature in different physical situations. For instance, with contact interacting repulsive bosons [11, 12, and with attractive interactions [13]; the effects of dipolar interaction [14, 15, as well as two distinct atomic species [12]. Moreover, the effects of a tunable tunneling in one ring has been also addressed [16, 17]. Quantized vortices, which are char-

\footnotetext{
* albert.escriva@fqa.ub.edu
}

acteristic flux states of superfluid systems in closed geometries, have been also explored in a ring lattice, see Refs. [16 22] and references therein.

In this paper we consider a primary integrated atomtronic circuit composed by two identical rings linearly coupled forming a two-ring ladder. In particular, we investigate two identical trimers coupled by an inter-ring tunneling parameter, that can be different from the intraring tunneling between neighboring sites in the same ring. We identify the effects arising from the competition among interactions and the two tunnel couplings in order to show that this is a flexible configuration that can be used for quantum technologies. By properly tuning interactions and tunneling strengths, in fact, the system ground-state is shown to approach different notable configurations, which, in turn, constitute prototypical atomtronic devices: from two stacked rings, to two fully disconnected rings or three disconnected double-wells, as well as all the intermediate situations.

The two-stacked-ring geometry is at the base of experimentally-available implementations of a matterwave based qubit system [23, 24. In these platforms, which combine the long coherence lifetime of neutral cold atoms systems with the robustness of topologically protected solid state Josephson flux qubits [25], it has been shown that the associated imaginary-time effective action provides a two-level-system dynamics for the phase slip across the two rings [23]. From the point of view of manybody physics, in the presence of a synthetic magnetic field, weakly- and strongly-interacting bosons on twoleg-ladder geometries are well known to disclose intriguing phase diagrams and complex magnetic-like phenomena, where circulating currents can exhibit characteristic Meissner-like and vortex-like patterns [26 34, reminiscent of the field dependence of currents in type-II superconductors [35]. In addition, it is worth mentioning 
that a rich dynamical scenario emerges if one focuses on the possible transfer of persistent currents and vortices between linearly coupled rings [36 43].

When the inter-ring tunneling is negligible with respect to the intra-ring hopping, the two rings are effectively decoupled. The resulting single-ring system has been subject of extensive study [5], as it can support the realization of a supercurrent-based qubit [24, 44]. In the opposite limit, that means when it is the intra-ring tunneling to be negligible with respect to the inter-ring tunneling, one remains with many decoupled double-well systems. The latter constitute the fundamental building block of several atomtronic devices 45 49, as it is well known that a Bose-Einstein condensate of neutral atoms in a double-well potential represents the matter-wave counterpart of a Josephson junction of coupled superconductors [5].

Depending on the relative value of its parameters, the model we investigate thus interpolates among different prototypical atomtronic systems and can hence capture not only standard "asymptotic limits", but also interesting cross-over regimes, where the competition among different couplings is at its most crucial. The aim of this paper is hence to provide an exhaustive characterization of the ground state properties of two coupled rings, in a wide range of tunneling strengths and interactions. We use exact diagonalization techniques to characterize the ground state properties of the two-ring bosonic ladder as a function of the tunneling parameters and onsite interaction strength for different number of atoms. For instance, we investigate fragmentation, entanglement and quantum correlations properties of this elemental integrated circuit that can help to guide future applications in atomtronics technologies. We pay special attention to the commensurability or incommensurability of the number of atoms with respect to the total number of sites of the system. We discuss the main differences, and we show that they could be used to indirectly determine whether the number of trapped bosons is even or odd. For instance, when the number of atoms is odd, the two rings remain always entangled for any range of onsite interactions. We also provide analytical expressions in the limit of large interactions in the case of non commensurate number of atoms. These characteristic features of a quantum system are particularly relevant as recent experiments are already able to measure entanglement properties [50] and quantum correlations in these setups [51, 52].

Our paper is organized as follows: In Sect. II we introduce the model Bose-Hubbard (BH) Hamiltonian as well as the parameters of the physical system. In Sect. III we present the analytical solutions of our model for the single-particle case. We discuss also the properties of the eigenstates of the non-interacting system and the lowlying energy spectrum obtained numerically. In Sect. IV we consider $N$ bosons loaded in the two-ring lattice and, by numerical diagonalization of the Hamiltonian, we investigate the static properties of the ground state of the two-coupled rings for different values of $N$, as well as the effects of the non commensurability of the number of atoms with respect to the number of sites, such as fragmentation, correlations between different sites, Schmidt gap and the entanglement entropy. Finally, in Sect. V]we provide the main conclusions of our work.

\section{THEORETICAL MODEL}

We consider $N$ bosons loaded in two stacked BoseHubbard rings, with the same number of sites $M$ in each ring and tunnel coupled via the rungs, see Fig. 1 for a schematic representation. The system is described by the following Hamiltonian,

$$
\begin{aligned}
\hat{\mathcal{H}} & =-J \sum_{j=\uparrow, \downarrow} \sum_{i=1}^{M}\left(\hat{a}_{i, j}^{\dagger} \hat{a}_{i+1, j}+\hat{a}_{i+1, j}^{\dagger} \hat{a}_{i, j}\right) \\
& -J_{\perp} \sum_{i=1}^{M}\left(\hat{a}_{i, \uparrow}^{\dagger} \hat{a}_{i, \downarrow}+\hat{a}_{i, \downarrow}^{\dagger} \hat{a}_{i, \uparrow}\right)+\frac{U}{2} \sum_{j=\uparrow, \downarrow} \sum_{i=1}^{M} \hat{n}_{i, j}\left(\hat{n}_{i, j}-1\right),
\end{aligned}
$$

where $J$ and $J_{\perp}$ are the tunneling parameters between neighboring sites in the same ring, and between the two rings, respectively. The bosonic creation (annihilation) operators $\hat{a}_{i, j}^{\dagger}\left(\hat{a}_{i, j}\right)$ for the site $i$ in the ring $j$ fulfil the canonical commutation relations, $\left[\hat{a}_{i, j}, \hat{a}_{k, l}^{\dagger}\right]=\delta_{i k} \delta_{j l}$. We introduce also the usual particle number operators $\hat{n}_{i, j}=\hat{a}_{i, j}^{\dagger} \hat{a}_{i, j}$ of the $i$ th site of the ring $j . U$ sets the strength of the atom-atom interaction which is assumed to be repulsive $(U>0)$.

This coupled system can be interpreted as a two-leg Bose-Hubbard ladder with periodic boundary conditions forming two connected rings with $M$ sites each one. In the ladder geometry, $J$ and $J_{\perp}$ correspond to the hopping along the rings and legs of the ladder. The competition between the two tunneling parameters determines the static properties of the two connected discrete rings. In particular, there are two limiting cases: when $J / J_{\perp} \rightarrow 0$ the system behaves as $M$ independent bosonic Josephson junctions, with two sites coupled by $J_{\perp}$ [53, whereas when $J / J_{\perp} \rightarrow \infty$ the two rings become fully decoupled. Many-body properties of a single lattice ring with finite number of sites loaded with contact interacting bosons, have been studied in Refs. [11, 13]. A general case of one ring with a tunable tunneling, has been previously studied for $M=3$ [16] and for an arbitrary (but small) number of sites [17]. In this work we consider mainly the minimal coupled atromtronic circuits; two stacked trimers.

\section{NON-INTERACTING LIMIT}

In this section we revisit the non-interacting limit of Hamiltonian (1), which admits analytical solutions. We will focus on the case $M=3$, which constitutes the minimal two-ring configuration. 


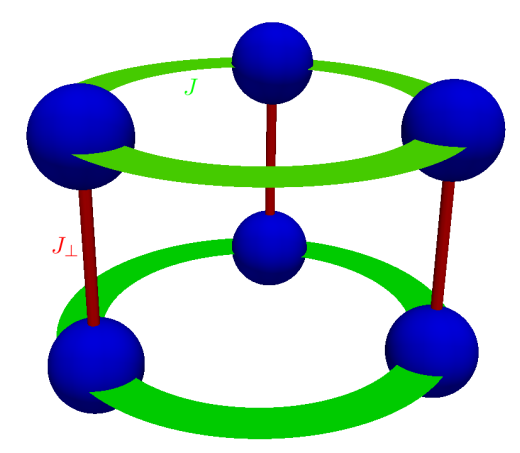

FIG. 1. Schematic representation of the minimal system with two stacked trimers. The intra-ring tunneling between the sites of the same ring is given by $J$ and the inter-ring tunneling between sites of different rings is given by $J_{\perp}$.

The non-interacting solutions of two linearly coupled trimers can be constructed as,

$$
\left|\Psi_{q}^{ \pm}\right\rangle=\frac{1}{\sqrt{2 M}} \sum_{l=1}^{M} e^{i \frac{2 \pi q l}{M}}\left(\hat{a}_{l, \uparrow}^{\dagger} \pm \hat{a}_{l, \downarrow}^{\dagger}\right)|\mathrm{vac}\rangle
$$

where $\mid$ vac $\rangle$ stands for the vacuum, $\hat{a}_{l}^{\dagger}$ is the creation operator of one atom in the $l$ site, and $q=0,1, \ldots, M-1$ labels the vortex wave function with quantization $2 \pi q$.

This solution was also found in Ref. [54] but in the continuous limit for an infinite number of sites by diagonalizing the Hamiltonian in momentum space by means of a Bogoliubov transformation. It is interesting to note that for the single-particle case, the eigenstates of two-coupled rings are independent of the tunneling parameters $J$ and $J_{\perp}$, and they involve only the same kind of flux state in both rings simultaneously. This combination can be either symmetric or antisymmetric. This means that a state formed by a combination of a vortex in the top ring and an antivortex in the bottom ring is not an eigenstate of the non-interacting two-coupled ring system.

In Ref. 42 we have studied linear combinations of noninteracting degenerate states, when $J_{\perp}=0$, that lead to single-particle vortices and fractional vortices. These states correspond to population-imbalanced vortices with current localized in one ring, and to population-balanced fractional vortices, respectively. They are stationary in the noninteracting limit of decoupled rings.

In Fig. 2 we show the single-particle energy spectrum for two stacked trimers as a function of the ratio between the inter-ring and intra-ring couplings $J_{\perp} / J$. The ground state is non degenerate for all values of the tunneling except for $J_{\perp}=0$, when there is no coupling between the two rings. In this case the two rings are independent and therefore the symmetric and antisymmetric combinations have the same energy. When $J_{\perp} / J>0\left(J_{\perp} / J<0\right)$ the ground state is the symmetric (antisymmetric) combination of the single ring ground state solutions with energies $\varepsilon_{0}^{+}=-J\left(J_{\perp} / J+2\right)$ and $\varepsilon_{0}^{-}=J\left(J_{\perp} / J-2\right)$, respectively. The symmetric

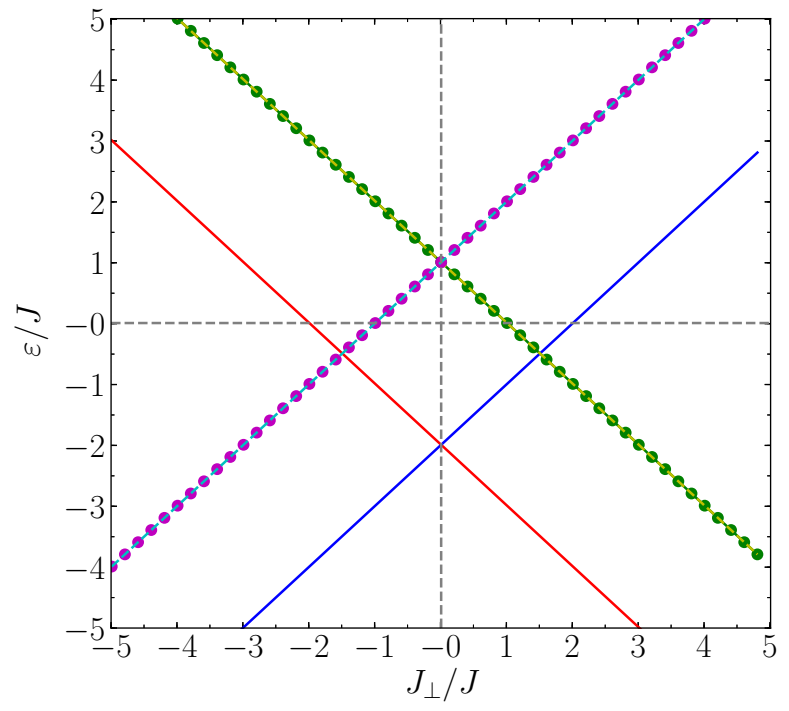

FIG. 2. Energy spectrum of two coupled trimers in the singleparticle case as a function of $J_{\perp} / J$. Red line corresponds to $\varepsilon_{0}^{+}$, blue line $\varepsilon_{0}^{-}$, green-dotted line $\varepsilon_{\mathrm{v}}^{+}$, yellow-dashed line $\varepsilon_{\mathrm{av}}^{+}$, magenta-dotted line $\varepsilon_{\mathrm{v}}^{-}$and blue-dashed line $\varepsilon_{\mathrm{av}}^{-}$.

and antisymmetric excited states present a double degeneracy corresponding to vortex-vortex and antivortexantivortex combinations: $\varepsilon_{\mathrm{v}}^{+}=\varepsilon_{\mathrm{av}}^{+}=J\left(1-J_{\perp} / J\right)$ and $\varepsilon_{\mathrm{v}}^{-}=\varepsilon_{\mathrm{av}}^{-}=J\left(1+J_{\perp} / J\right)$, where we have used the notation $q=0$ (gs), $1(\mathrm{v}),-1$ (av). The energy spectrum is symmetric with respect to the change of sign of $J_{\perp} / J$.

In order to study the static properties of our system for different values of the interaction and tunneling parameters, we calculate the ground state and the lower part of the energy spectrum by numerical diagonalization of the Hamiltonian [55, 56, for different numbers of atoms.

In Fig. 3 we plot the energy spectrum, for different values of the interaction $U / J_{\perp}$, where $k$ is the spectral index. We observe the existence of energy bands for small values of the interaction, which breakdown when the interaction rate is increased. As explained in Ref. [17] the band structure can be understood by means of the number of atoms and the degeneracy of the flow basis.

\section{GROUND STATE PROPERTIES OF THE INTERACTING SYSTEM}

The system formed by $N$ interacting bosons loaded in two coupled atomtronic circuits is described by the Hamiltonian (1) and has three physical parameters: the interatomic interaction $U$, and the tunneling strengths $J$ and $J_{\perp}$. In modern experimental setups, these parameters can be tuned in a very controlled way [24, 57, although their specific variability ranges strictly depend on the chemical element which is Bose-condensed and on the optical apparatus which is employed. In Ref. [58, 


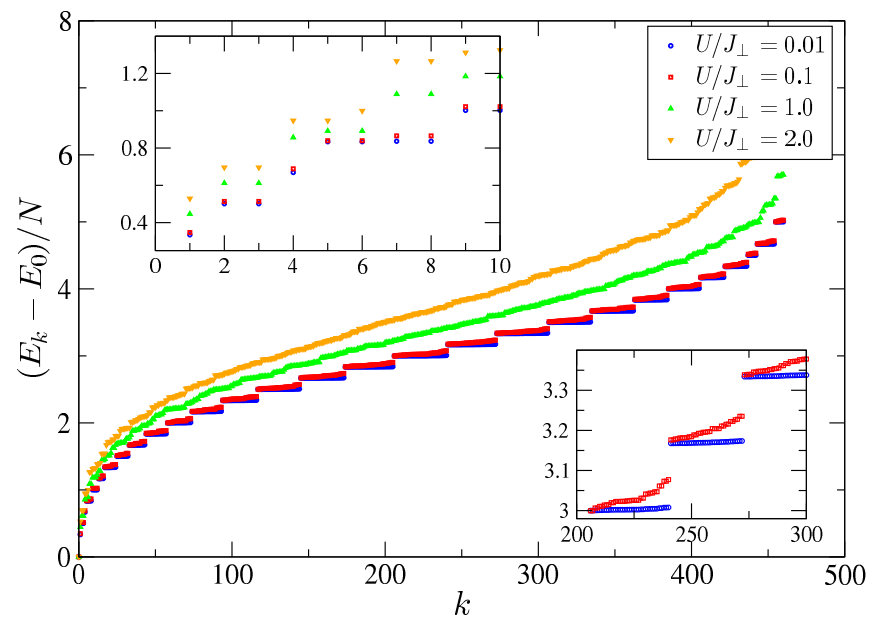

FIG. 3. Excitation energies per particle, $\left(E_{k}-E_{0}\right) / N$ of the system with $N=6, M=3$ and $J / J_{\perp}=1.0$, for different values of $U / J_{\perp}$.

for example, the experimental realization of a bosonic two-leg ladder in the weakly interacting regime, meaning that $U / J_{\perp} \approx 0$, was reported. In this remarkable experiment, measurements were performed in the range $J_{\perp} / J \in[0,3.5]$, i.e. from a regime where the two rings are decoupled to a regime where the inter-ring coupling is 3.5 times larger than the intra-ring coupling. In Ref. 35, the complementary regime was explored, i.e. the strongly interacting one (which was claimed to be within the reach of available experimental setups). Numerical simulations were performed in a rather extended range of model parameters, i.e. $U / J \in[0,+\infty)$ and $J_{\perp} / J \in[0,8]$.

In this section, we characterize the ground state of the two-connected trimers by means of coherence and fragmentation properties, correlations between different sites, as well as the entanglement between the two rings. We will discuss also the effect of incommensurate filling.

\section{A. Limiting situations}

Let us recall the analytical solutions of the ground state in two limiting cases. When the tunneling terms dominate $\left(U / J \rightarrow 0\right.$ and $\left.U / J_{\perp} \rightarrow 0\right)$, this leads to a complete delocalization of each atom over all the sites. The noninteracting limit in the homogeneous case corresponds to the superfluid phase (Bose-Einstein condensate, BEC). In our discrete system, the coherent ground state reads,

$$
\left|\Psi_{\mathrm{BEC}}\right\rangle=\frac{1}{\sqrt{N !}}\left(\frac{1}{\sqrt{2 M}} \sum_{j=\uparrow, \downarrow} \sum_{i=1}^{M} \hat{a}_{i, j}^{\dagger}\right)^{N}|\mathrm{vac}\rangle .
$$

When the repulsive interaction dominates $(J / U \rightarrow 0$ and $J_{\perp} / U \rightarrow 0$, with $U>0$ ) the tunneling energies become small and the atoms tend to localize. Therefore, the system prefers to reduce the number of pairs in each site to minimize the energy. The ground state has equipopulation with, on average, $\nu=N /(2 M)$ atoms on each site, when $\nu \in \mathbb{Z}$. The strong interacting limit of one ring with incommensurate filling (non integer filling factor) has been studied in Ref. [11. Here we start the analytical study to commensurate number of atoms, but we also discuss the ground state properties of two coupled rings when one atom is added (or subtracted) from a commensurate case. The localized ground state for a commensurate system (Mott insulator phase, MI, in the homogenous system) corresponds to one state of the Fock basis:

$$
\left|\Psi_{\mathrm{MI}, \nu}\right\rangle=\prod_{j=\uparrow, \downarrow} \prod_{i=1}^{M} \frac{\left(\hat{a}_{i, j}^{\dagger}\right)^{\nu}}{\sqrt{\nu !}}|\mathrm{vac}\rangle
$$

assuming that the number of atoms $N$ is commensurate with the number of sites $2 M, \nu=N /(2 M) \in \mathbb{Z}$. When one atom is added (or subtracted) the system has $N \pm 1$ atoms with filling factor $\nu^{ \pm}=(N \pm 1) /(2 M)=\nu \pm$ $1 /(2 M)$. The ground state of the resulting system is, respectively:

$$
\begin{aligned}
& \left|\Psi_{\mathrm{MI}, \nu^{+}}\right\rangle=\frac{1}{\sqrt{2 M}} \frac{1}{\sqrt{\nu+1}} \sum_{j=\uparrow, \downarrow} \sum_{i=1}^{M} \hat{a}_{i, j}^{\dagger}\left|\Psi_{\mathrm{MI}, \nu}\right\rangle, \\
& \left|\Psi_{\mathrm{MI}, \nu^{-}}\right\rangle=\frac{1}{\sqrt{2 M}} \frac{1}{\sqrt{\nu}} \sum_{j=\uparrow, \downarrow} \sum_{i=1}^{M} \hat{a}_{i, j}\left|\Psi_{\mathrm{MI}, \nu}\right\rangle .
\end{aligned}
$$

These two states can be obtained by distributing an extra particle (or vacancy) between the different sites of the commensurate state (4).

\section{B. Coherence and fragmentation}

We investigate the coherence of the system by analyzing the condensed fraction and fragmentation properties which are defined by the eigenvalues of the one-body density matrix [59]. For a many-body state $|\Psi\rangle$ describing $N$ bosons in $2 M$ sites (two rings with $M$ sites), the onebody density matrix, $\hat{\rho}$, is a $2 M \times 2 M$ matrix, whose elements read

$$
\rho_{(i, j),(k, l)}=\frac{1}{N}\left\langle\Psi\left|\hat{a}_{i, j}^{\dagger} \hat{a}_{k, l}\right| \Psi\right\rangle
$$

where $i, k=1, \ldots, M$ and $j, l=\uparrow, \downarrow$. Since $|\Psi\rangle$ is normalized to one, this implies that $\operatorname{Tr}(\hat{\rho})=1$. The eigenvalues of the one-body density matrix are the relative occupation numbers, $p_{i}=N_{i} / N$, of the corresponding eigenvectors (single-particle states or natural orbitals). They are normalized to 1 , meaning that $p_{1}+p_{2}+. .+p_{2 M}=1$, and they are conventionally labelled in such a way that $p_{1} \geq p_{2} \geq . . \geq p_{2 M} \geq 0$ [59]. In the case of a singly condensed system there is only one large eigenvalue $p_{1} \approx 1$ and the others are very small $p_{i} \sim \mathcal{O}(1 / N)(i \neq 1)$. This means that there is a macroscopic occupation of the corresponding single-particle state $\sim \mathcal{O}(N)$ and the 


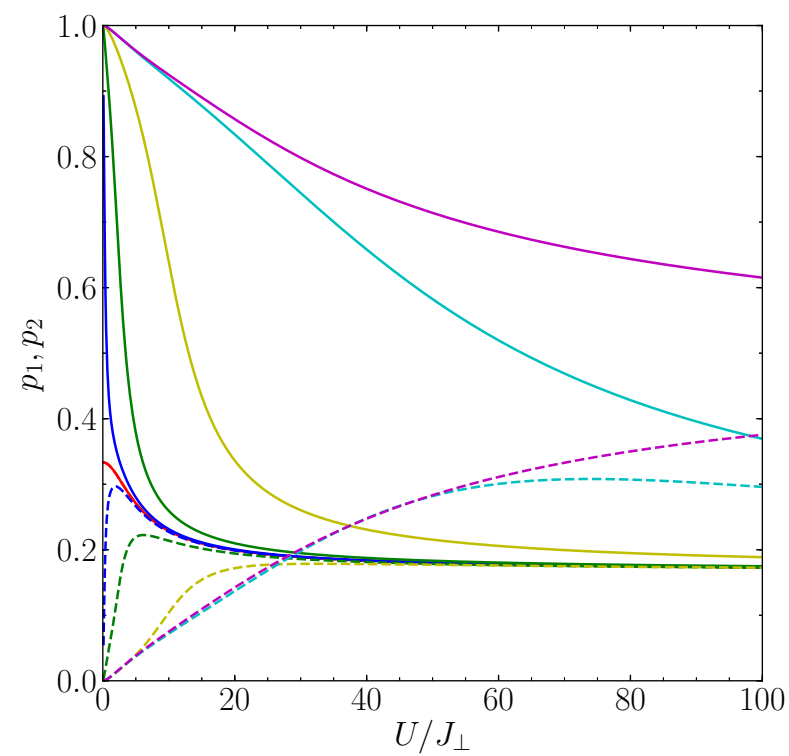

FIG. 4. The two largest eigenvalues of the one-body density matrix, $p_{1}$ (solid) and $p_{2}$ (dashed), as a function of $U / J_{\perp}$ for different values of $J / J_{\perp}$. In all cases: $N=6$ and $M=3$. Color legend (from bottom to top): $\operatorname{red}\left(J / J_{\perp}=0, p_{1}\right.$ and $p_{2}$ are overlapped), blue $\left(J / J_{\perp}=10^{-2}\right)$, green $\left(J / J_{\perp}=10^{-1}\right)$, yellow $\left(J / J_{\perp}=1\right)$, cyan $\left(J / J_{\perp}=10^{1}\right)$ and magenta $\left(J / J_{\perp}=\right.$ $\left.10^{2}\right)$.

system is condensed (it is worth stressing that the macroscopic occupation of a natural orbital is a typical feature of Bose-Einstein condensation and it is what supports the possibility of introducing a "macroscopic wave function" for the system [59]. Whereas when there is more than one large eigenvalue, the system is fragmented [59]. The ground state with largest fragmentation corresponds to all eigenvalues $p_{i}=1 /(2 M)$ in the Mott insulator limit.

We remark that coherence is a crucial ingredient in the design of atomtronic circuits [5, 60, while fragmentation tends to hinder their operation. When the two-ring ladder is used as a superfluid qubit system [24, condensate's fragmentation tends to destroy the topologically protected quantum state (which realizes the qubit itself) and should therefore be minimized. In addition, fragmentation, which is inevitably present in interacting systems, can lead to the suppression of the coherent transfer of vorticity between the rings 42 .

In Fig. 4 we show the two largest relative occupation numbers, $p_{1}$ and $p_{2}$, of two coupled trimers as a function of $U / J_{\perp}$, with $N=6$ and for different values of $J / J_{\perp}$. For weak inter-particle interactions $\left(U \ll J_{\perp}\right)$ there is a non-zero single particle state, $p_{1} \simeq 1$, for all values of $J / J_{\perp}$ except when $J / J_{\perp}=0$. In the latter case, the sites in the same ring are fully decoupled and the system reduces to three independent double-wells with tunneling rate $J_{\perp}$. Thus, a three-fragmented state with $p_{1}=p_{2}=p_{3}=1 / 3$ is obtained when $J / J_{\perp}=0$ and
$U / J_{\perp} \lesssim 1$. In this case the total wave function is not given by Eq. (3), but by a product state of three independent bosonic Josephson junctions with $N / M$ particles in each junction, when $N / M \in \mathbb{Z}$.

As the interaction increases and the ratio $U / J_{\perp}$ becomes large, the atoms start to localize and the system tends to a Mott-insulator-like phase in the asymptotic limit where the ground state is fully fragmented and $p_{i}=1 /(2 M)=1 / 6$. However, due to the particular geometry of our system with two competing tunneling rates $J$ and $J_{\perp}$, there are two different regimes, $J>J_{\perp}$ and $J_{\perp}<J$, which clearly appear in the asymptotic values of Fig. 4 . When $J / J_{\perp} \leq 1$ the ground state reaches a fragmented state for smaller values of $U / J_{\perp}$ than when $J / J_{\perp}>1$. For a fixed value of $U / J_{\perp}$, when the intraring tunneling is dominant in front of the inter-ring coupling $\left(J>J_{\perp}\right)$, the system is less fragmented than for $J / J_{\perp}<1$. This follows from the condition $\sum_{i}^{2 M} p_{i}=1$. Figure 4 shows that $p_{1}+p_{2} \simeq 1$ when $J>J_{\perp}$, whereas when $J<J_{\perp}$ all the eigenvalues of the one-body density matrix $p_{i} \neq 0$ which corresponds to a larger fragmentation of the ground state. The system we are considering has $2 M$ intra-ring couplings $J$ (that is, $2 M$ pairs of sites connected by $J$ ) and $M$ inter-ring couplings $J_{\perp}$ ( $M$ pairs of sites connected by $J_{\perp}$ ). Thus, when $J>J_{\perp}$ the tunneling effects are larger than when $J<J_{\perp}$, and the ground state remains delocalized for larger values of $U / J_{\perp}$. The system needs larger interactions to balance the delocalization promoted by the tunneling. A similar reasoning holds also if one increases the (commensurate) number of atoms $N$. As is well known, in fact, higher (integer) fillings correspond to smaller Mott lobes and, therefore, to systems which undergo Mott localization for larger values of the interaction $U$ 61]. Hence, upon increasing the (integer) filling $\nu$, the behaviour illustrated in Fig. 4 is qualitatively unchanged, although the asymptotic value $p_{1}=p_{2}=1 /(2 M)$ is reached for larger values of $U$.

A complementary information is provided by its corresponding von Neumann entropy, which measures the condensation of the system. It is defined from the eigenvalues of the one-body density matrix as

$$
S_{\mathrm{VN}}=-\sum_{i=1}^{2 M} p_{i} \ln p_{i}
$$

It is a bounded quantity: the minimum value is $S_{\mathrm{VN}}=0$ and corresponds to a fully condensed ground state $\left(p_{1}=1\right)$, whereas the maximum value of $S_{\mathrm{VN}}$ is $\ln (2 M)$ and occurs for a completely fragmented state with $p_{i}=$ $1 /(2 M)$, for all $i$. When $S_{\mathrm{VN}}=\ln s$ (with $s \in \mathbb{Z}^{+}$) the system is fragmented in $s$ states.

Figure 5 presents $S_{\mathrm{VN}}$ for the two stacked trimers with $N=6$ atoms as a function of $U / J_{\perp}$, for the same cases $J / J_{\perp}$ as in Fig. 4. One can see that for a fixed value of $U / J_{\perp}$, the entropy decreases as $J / J_{\perp}$ increases; that is, the ground state is less fragmented when $J>J_{\perp}$ than when $J<J_{\perp}$, as we have already obtained in Fig. 4. In 


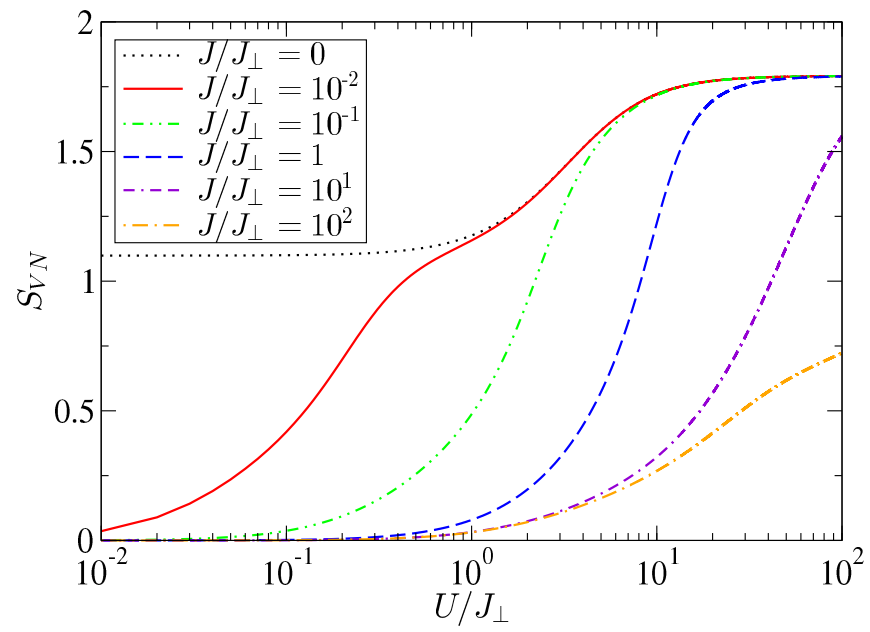

FIG. 5. Von Neumann entropy of the one body density matrix as a function of $U / J_{\perp}$ for different values of $J / J_{\perp}$. In all cases, $N=6$ and $M=3$.

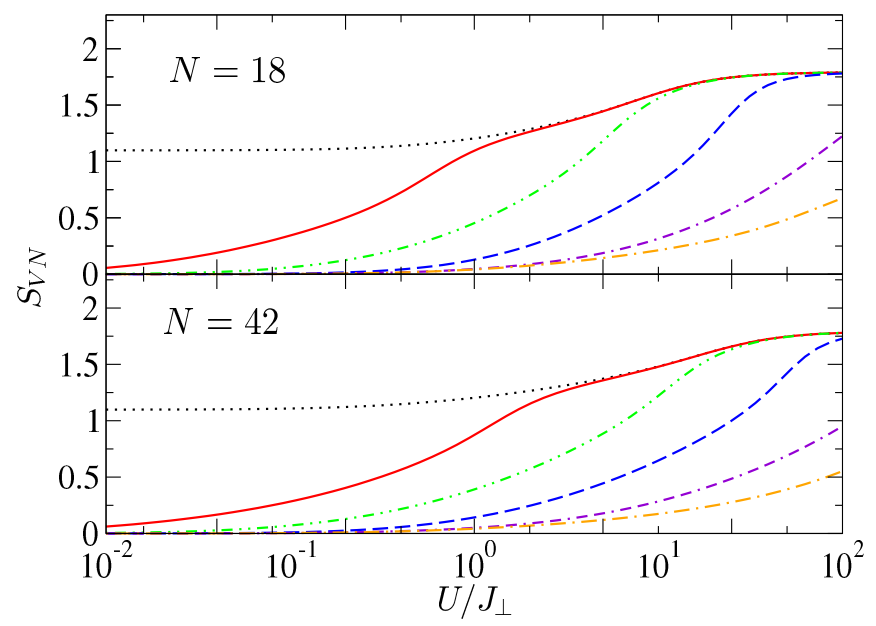

FIG. 6. Von Neumann entropy of the one body density matrix as a function of $U / J_{\perp}$ for different values of $J / J_{\perp}$ and particles $N$ : top panel $(N=18)$, bottom panel $(N=42)$. In all cases $M=3$.

the limiting case $J=0$ and when $U / J_{\perp}>1$ the entropy is given by $S_{\mathrm{VN}}=\ln M=\ln 3$, since the system behaves as $M=3$ bosonic Josephson junctions.

When $J / J_{\perp} \geq 0.1$ and for small values of $U / J_{\perp}$, the ground state corresponds to a coherent state and $S_{\mathrm{VN}} \simeq$ 0 . From Fig. 5 one can see that this regime in terms of $U / J_{\perp}$ broadens when $J>J_{\perp}$. For large interactions the system tends to the hard-core boson limit with a $2 M$ fragmented state, whose entropy is $S_{\mathrm{VN}}=\ln (2 M)=\ln 6$. This regime is achieved for smaller values of $U / J_{\perp}$ when $J<J_{\perp}$. The localization of the particles in different sites requires larger interaction strengths, $U / J_{\perp}>10^{2}$ when $J / J_{\perp}>1$, as we have commented in the previous figure.

We have extended the analysis of the condensation and fragmentation properties for larger and commensurate number of particles confined in the two coupled rings.
The results are summarized in Figs. 6 and 7 , where we plot the von Neumann entropy and the eigenvalues of the one-body density matrix, respectively, as a function of $U / J_{\perp}$ for $N=18,42$ atoms, and $M=3$. In general, the same physical behavior is obtained for all the cases we have studied. In Fig. 6 one can see that the limiting values of $S_{\mathrm{VN}}$ do not depend on the number of atoms confined in the two connected rings. As in the previous case with $N=6$ atoms, independently of the number of commensurate atoms, for small interactions the system is three-fragmented in three disconnected double-wells when $J / J_{\perp}=0$, whereas for $J / J_{\perp} \neq 0$ the system is condensed. In the other limit, for large interactions the system tends to the Mott insulator regime more rapidly when $J / J_{\perp} \leq 1$. As shown in Fig. 6, this asymptotic Mott insulator limit, $S_{\mathrm{VN}}=\ln (2 M)$, when $J / J_{\perp}>1$ is reached for larger values of $U / J_{\perp}$ when the number of atoms increases. Similarly, for a given ratio $J / J_{\perp}$, upon increasing the (integer) filling $\nu$, the behaviour of the Von Neumann Entropy is qualitatively unchanged, although the asymptotic value $S_{\mathrm{VN}}=\ln (2 M)=\ln 6$ is reached for larger values of $U$. This property clearly emerges from the comparison of Fig. 5 and both panels of Fig. 6 .

The eigenvalues of the one-body density matrix for $N=18$ (top panels), and 42 (bottom panels) are plotted in Fig. 7 for different values of the tunneling ratios: $J / J_{\perp}=0$ (left panels), $J / J_{\perp}=1$ (middle vertical panels) and $J / J_{\perp}=10$ (right panels). When $J / J_{\perp}=0$, since the sites in the same ring are decoupled, the system behaves as three independent double-wells and therefore $p_{1}=p_{2}=p_{3}$ and $p_{4}=p_{5}=p_{6}$ for any value of $U / J_{\perp}$. For small interactions the system is condensed in the double ring $\left(p_{1} \simeq 1\right)$ when $J / J_{\perp} \neq 0$, or in three double-wells $\left(p_{1}=p_{2}=p_{3}=1 / 3\right)$ when $J / J_{\perp}=0$. As before, for large interactions the system tends to the Mott insulator regime $p_{i}=1 /(2 M)(i=1,2, \ldots, 6)$. This limit is achieved for smaller interactions $U / J_{\perp}$, when $J / J_{\perp} \leq 1$ for a fixed number of atoms, or for smaller number of commensurate atoms when the tunneling ratio is fixed.

\section{Incommensurate number of atoms}

We have also investigated the coherence and fragmentation properties of the system when an incommensurate number of atoms (with respect to the total number of sites $2 M$ ) is trapped in the double ring. Starting from the commensurate situation with $N$ atoms, we have considered the case with one added (or subtracted) extra particle, $(N+1)$ and $(N-1)$ atoms, respectively.

Figure 8 shows the eigenvalues of the one-body density matrix (top panel) and its corresponding von Neumann entropy (bottom panel), as a function of $U / J_{\perp}$, obtained numerically by solving the Hamiltonian (1) for two-connected rings, with $M=3$ and 19 atoms. This situation corresponds to adding one extra particle from the $N=18$ commensurate case studied previously with filling factor $\nu=3$. For small interactions, $U / J_{\perp}<1$, 


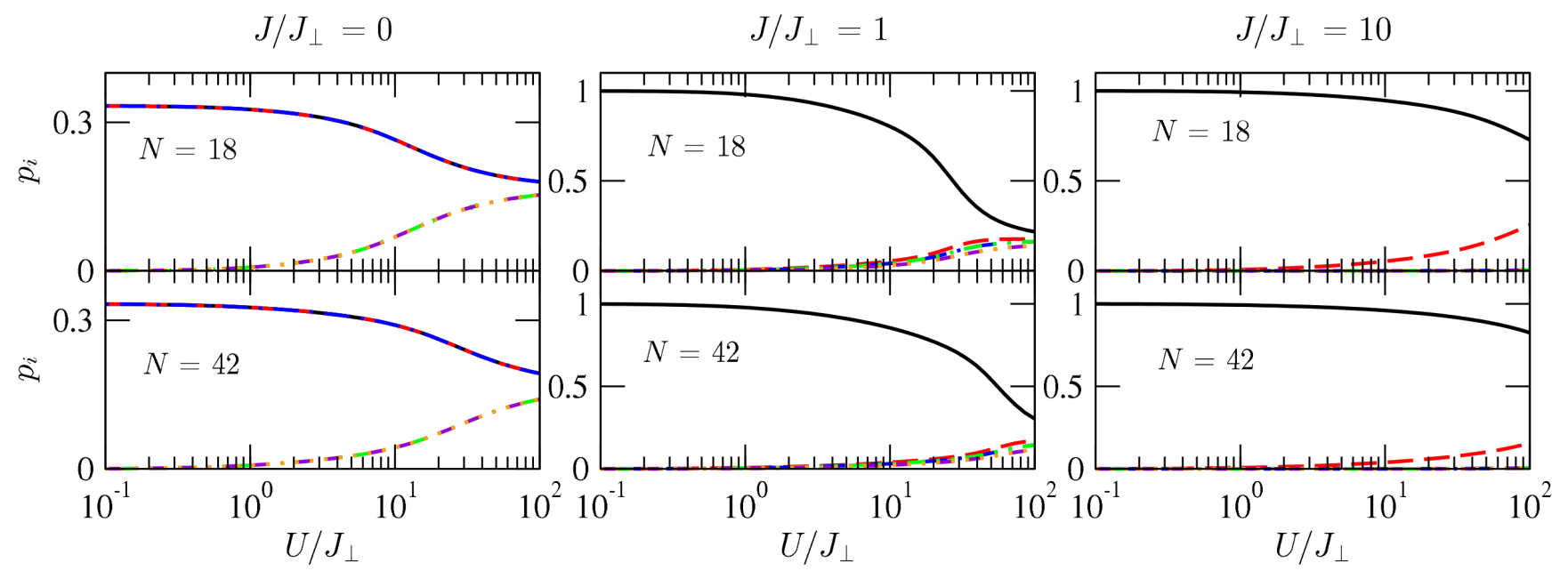

FIG. 7. Eigenvalues of the one-body density matrix as a function of $U / J_{\perp}$ (in logarithmic scale) for different values of $J / J_{\perp}$, and $N$. Top panels correspond to $N=18$, and bottom ones to $N=42$. Left panels correspond to $J / J_{\perp}=0$, middle panels to $J / J_{\perp}=1$ and right panels to $J / J_{\perp}=10$. Black solid line represents the largest eigenvalue $p_{1}$. The other eigenvalues $p_{i}$ are shown with dashed colored lines: red $p_{2}$, blue $p_{3}$, green $p_{4}$, violet $p_{5}$ and orange $p_{6}$.

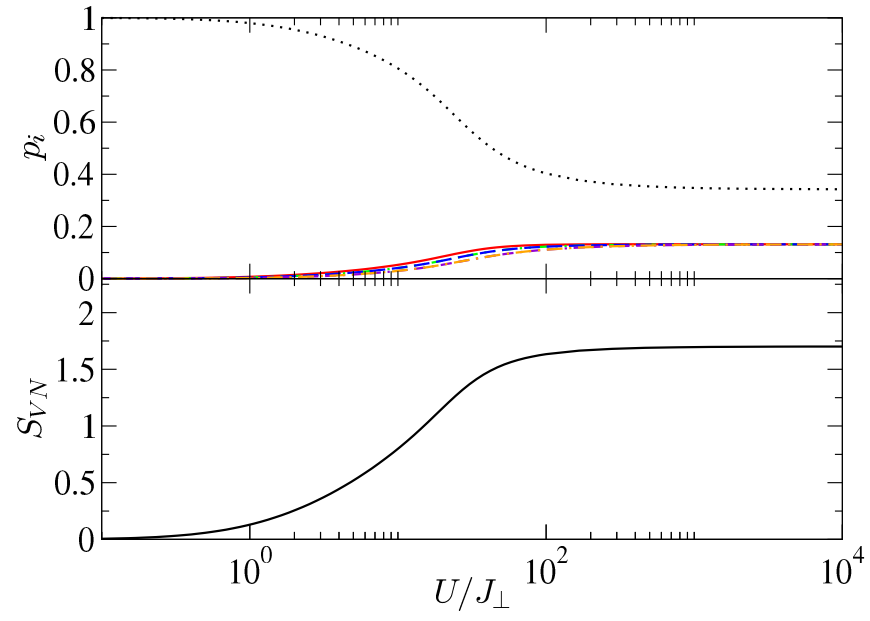

FIG. 8. Eigenvalues of the one-body density matrix (upper panel) and von Neumann entropy (lower panel), as a function of $U / J_{\perp}$ for $J / J_{\perp}=1$ computed with 19 atoms and $M=3$. Top panel: black dotted line represents the largest eigenvalue $p_{1}$, the other colored lines represent the other eigenvalues.

the ground state properties are almost the same with $N$ or $N+1$ atoms, and the ground state behaves as in the commensurate case: $S_{\mathrm{VN}} \simeq 0, p_{1} \simeq 1$, and $p_{j} \simeq 0$ for $j \neq 1$. However, for large interactions $U / J_{\perp}>10^{2}$, when one atom is added, the system is not fully fragmented as in the commensurate situation where the atoms are localized in the sites with filling factor $\nu=N /(2 M)$. Here, the extra atom is not localized since it is shared with all the sites. Therefore the system cannot reach the fully fragmentation limit corresponding to the Mott insulator values $p_{i}=1 /(2 M)=1 / 6$ for all $i$, and $S_{\mathrm{VM}}=\ln (2 M)=\ln 6$.

In the large interaction limit, when one atom is added (subtracted) from the commensurate case, the analytical expressions of the corresponding one-body density matrix, $\hat{\rho}^{+}(\hat{\rho}-)$, and their eigenvalues, can be obtained from Eqs. (5) and (6). The diagonal matrix elements are given by $\rho_{(i, j)(i, l)}^{+}=\rho_{(i, j)(i, l)}^{-}=1 /(2 M)$, and the off-diagonal elements are $\rho_{(i, j)(k, l)}^{+}=(1 / N)(N-1+2 M) /(2 M)^{2}$ and $\rho_{(i, j)(k, l)}^{-}=(1 / N)(N+1) /(2 M)^{2}$. From them it follows the whole set of eigenvalues: the largest one is $p_{1}^{+}=\rho_{(i, j)(i, l)}^{+}+(2 M-1) \rho_{(i, j)(k, l)}^{+}$, and the others are $p_{j}^{+}=\rho_{(i, j)(i, l)}^{+}-\rho_{(i, j)(k, l)}^{+}$. The same expressions hold for the case of one subtracted atom, by changing the components $\rho_{(i, j)(k, l)}^{+}$by $\rho_{(i, j)(k, l)}^{-}$. These equations can be simplified by introducing the corresponding filling factor, which for one added extra particle is $\nu^{+}=\nu+1 /(2 M)$,

$$
p_{1}^{+}=\frac{4 \nu M+2 M-\nu}{4 \nu M^{2}+2 M}, \quad p_{j}^{+}=\frac{2 \nu M-\nu}{4 \nu M^{2}+2 M} .
$$

It is straightforward to prove that these expressions verify the eigenvalues relation of the one-body density matrix: $\sum_{i=1}^{2 M} p_{i}^{+}=p_{1}^{+}+(2 M-1) p_{j}^{+}=1$. Analogously for the case of one subtracted particle, the filling factor is $\nu^{-}=$ $\nu-1 /(2 M)$ and

$$
p_{1}^{-}=\frac{4 \nu M-\nu-1}{4 \nu M^{2}-2 M}, \quad p_{j}^{-}=\frac{2 \nu M-\nu-1}{4 \nu M^{2}-2 M} .
$$

In the limit of large interactions $U / J_{\perp} \gg 1$, the von Neumann entropy (7) can be also computed analytically from the corresponding eigenvalues of the one-body density matrix, Eqs. (8) or (9). In both cases $S_{\mathrm{VN}}^{+}=S_{\mathrm{VN}}^{-}$, since adding or subtracting one particle, i.e. having one extra particle or one vacancy, is equivalent from the entropic point of view. In the particular case of Fig. 8 , with $\nu=3$ and $18+1$ atoms, the limiting values for large interactions computed analytically from Eqs. (8) 
are: $p_{1}^{+}=13 / 38, p_{j,}^{+}=5 / 38$ for $j \neq 1$, and $S_{\mathrm{VN}}^{+}=1.726$, which are in agreement with the numerical results calculated from the Hamiltonian. In the incommensurate case with $18-1$ atoms, which corresponds to one subtracted atom from the commensurate system $N=18$, the corresponding values in the limit of large interactions are $p_{1}^{-}=16 / 51, p_{j}^{-}=7 / 51$, but the same entropy $S_{\mathrm{VN}}^{-}=1.726$ as adding one atom.

\section{Correlations}

In general, entropy-based indicators are rather versatile quantities allowing for the detection of quantum phase transitions in one-component [56] and multicomponent 62 66] Bose-Hubbard models. Here, we focus on the correlations between particles on different sites, and we quantify it by means of the entropic indicator [56]

$$
S_{\mathrm{C}}=-\sum_{\alpha}\left|c_{\alpha}\right|^{2} \ln \left|c_{\alpha}\right|^{2}
$$

where the term $c_{\alpha}$ is the coefficient of the Fock state $|\alpha\rangle$ in the expansion $|\Psi\rangle=\sum_{\alpha} c_{\alpha}|\alpha\rangle$. In essence, indicator $S_{\mathrm{C}}$ quantifies the degree of clustering of particles in the Fock space. The collection of all coefficients $\left|c_{\alpha}\right|^{2}$, in fact, can be regarded as a probability distribution over the space of Fock states (notice, also, that it is correctly normalized to 1 , since $\left.\sum_{\alpha}\left|c_{\alpha}\right|^{2}=1\right)$.

If only one Fock state, $|\beta\rangle$, enters in the linear combination above, as in the case of a fully fragmented state [59], then the probability distribution is maximally peaked $\left(\left|c_{\beta}\right|^{2}=1,\left|c_{\alpha}\right|^{2}=0, \forall \alpha \neq \beta\right)$, hence $S_{\mathrm{C}}=0$. Conversely, if two or more Fock states have non-zero projection on $|\Psi\rangle$, then the probability distribution $\left\{\left|c_{\alpha}\right|^{2}\right\}$ is broader and the associated entropy $S_{\mathrm{C}}$ is non-zero. For example, when the state of the system with $N=2 M$ atoms forms a single Bose-Einstein condensate, Eq. (3), the $S_{\mathrm{C}}$ can be calculated analytically as $S_{\mathrm{C}}=N \ln (2 M)-$ $(1 / 2 M)^{N} \sum_{n_{1}, \ldots, n_{2 M}}^{N}\left(\begin{array}{c}N \\ n_{1}, \ldots, n_{2 M}\end{array}\right) \ln \left(\begin{array}{c}N \\ n_{1}, \ldots, n_{2 M}\end{array}\right)$.

In Fig. 9 we plot $S_{\mathrm{C}}$ of the ground state of the twocoupled trimers, as a function of $U / J_{\perp}$ for the same values of $J / J_{\perp}$ as in Figs. 4 and 5 . As we have already seen in the previous figure, when $U \ll J, J_{\perp}$ the system is almost condensed, but $S_{\mathrm{C}}$ presents two limiting values: $S_{\mathrm{C}}=3.12$ when $J / J_{\perp}=0$, and $S_{\mathrm{C}}=5.76$ when $J / J_{\perp} \neq 0$. The latter value is in agreement with the previous analytical expression of $S_{\mathrm{C}}$ with $N=2 M=6$. It means that when $U / J_{\perp}<1$ and $J / J_{\perp} \neq 0$ the ground state corresponds to one Bose-Einstein condensate. Moreover, the system remains condensed for larger values of the interaction $U / J_{\perp}$ as the tunneling ratio $J / J_{\perp}$ increases. Another physical situation appears in the particular case when $J / J_{\perp}=0$; the system is decoupled into three independent bosonic Josephson junctions with tunneling rate $J_{\perp}$ and $N / M=2$ bosons in each one. In this case, when the ratio $U / J_{\perp}<1$, the bosons are

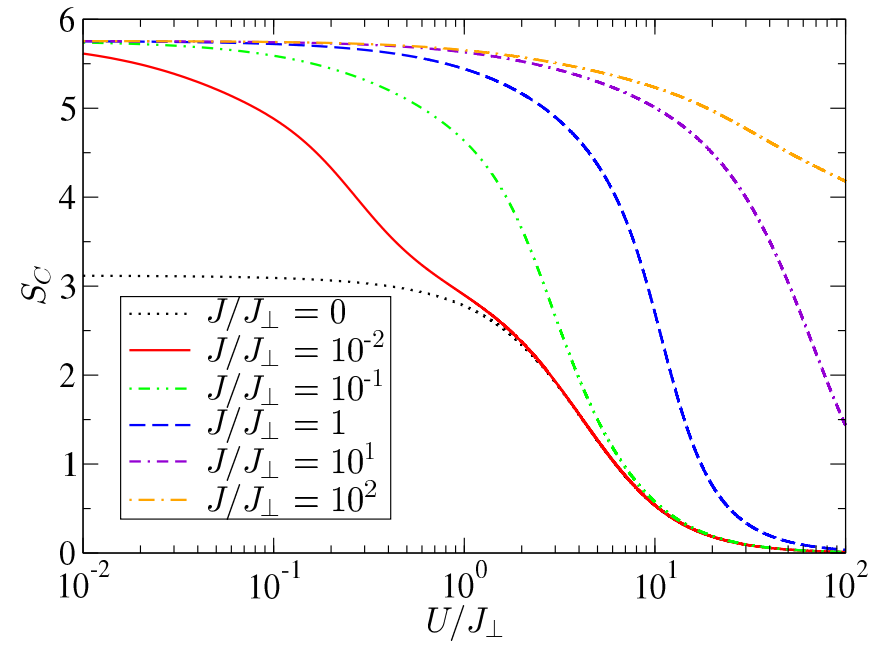

FIG. 9. $S_{\mathrm{C}}$ of the ground state as a function of $U / J_{\perp}$ for different values of $J / J_{\perp}$. In all cases, $N=6$ and $M=3$.

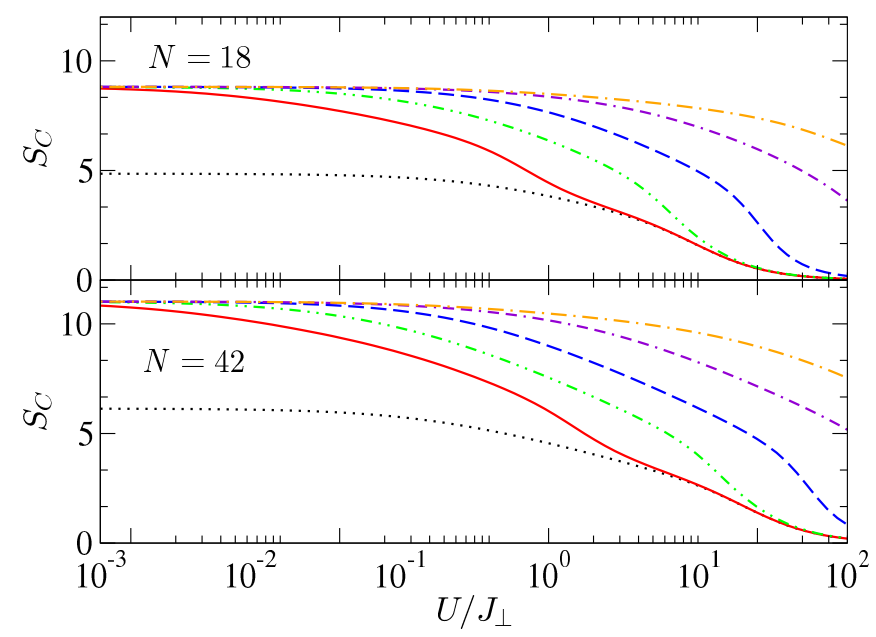

FIG. 10. $S_{C}$ of the ground state as a function of $U / J_{\perp}$ for different values of $J / J_{\perp}$ and number of particles: $N=18$ (top panel), and $N=42$ (bottom panel). In all cases $M=3$. The color legend is the same as in Fig. 9 .

occupying the same single-particle state of each Josephson junction. The entropy can be also computed analytically from the three independent double wells which yields $S_{\mathrm{C}}=3.12$ in agreement with our numerical results.

In the limit of large interactions $S_{\mathrm{C}}$ is given by $S_{\mathrm{C}} \rightarrow 0$ for all values of $J / J_{\perp}$; since in this situation the state of the system is fragmented and can be approximated by one Fock state with one particle localized in each site (Mott insulator regime). The largest localization with $S_{\mathrm{C}}=0$ is achieved for smaller values of the interaction $U / J_{\perp}$ as the tunneling ratio $J / J_{\perp}$ decreases. It is important to stress that if the number of particles is not commensurate with the total number of sites this yields $S_{\mathrm{C}} \neq 0$ in the Mott limit, since the ground state is not given by only one Fock state but by a superposition of Fock states obtained by adding the extra particles that 
are shared with all sites [11. In the particular case of one added (subtracted) atom from a commensurate system, $S_{C}^{+}\left(S_{C}^{-}\right)$in the large interaction (Mott) regime can be computed analytically using Eq. (5). For a given number of sites $M, S_{C}^{+}=S_{C}^{-}=\ln (1 / 2 M)$.

From Fig. 9 one can see that for a fixed value of $U / J_{\perp}$, the spatial correlation given by $S_{\mathrm{C}}$, increases for larger values of $J / J_{\perp}>1$ when the intra-ring coupling dominates over the inter-ring tunneling. This is in agreement with the results obtained from the previous quantities which indicate that the condensate fraction $p_{1}$ increases, and $p_{1}+p_{2} \simeq 1$, when $J / J_{\perp}>1$ increases. In this case the distribution of non-zero Fock coefficients of the ground state is larger and thus the spatial correlations. Concerning the behaviour of $S_{\mathrm{C}}$ in the large- $N$ limit, comparing Fig. 9 with both panels of Fig. 10, one can observe that, for a given value of $J / J_{\perp}$, the asymptotic limit $S_{\mathrm{C}}=0$ is approached for a larger value of $U / J_{\perp}$ if the (commensurate) number of bosons $N$ is larger.

To get further insight into the correlations of the particles one can compute the pair correlation function between two particular sites of the system. In general, the pair correlation between site $i$ (of ring $j$ ) and site $k$ of (ring $l$ ) is defined as,

$$
\eta_{(i, j),(k, l)}=\frac{\left\langle\Psi\left|\hat{a}_{i, j}^{\dagger} \hat{a}_{k, l}^{\dagger} \hat{a}_{i, j} \hat{a}_{k, l}\right| \Psi\right\rangle}{\rho_{(i, j),(i, j)} \rho_{(k, l),(k, l)}} .
$$

The value of $\eta_{(i, j),(k, l)}$ gives the probability of finding one particle in site $i$ (of ring $j$ ) when there is already a particle in site $k$ (of ring $l$ ). It is possible to write it in matrix form whose dimension is $2 M \times 2 M$, which contains all the pair correlations between all the sites. In our system composed by two stacked identical rings, one can distinguish two types of pair correlations: intra-ring correlations $\eta^{1 R} \equiv \eta_{(1, j),(2, j)}$ (correlations between two neighbor sites in the same ring coupled by $J$ ), and interring correlations $\eta^{2 R} \equiv \eta_{(i, \uparrow),(i, \downarrow)}$ (correlations between a pair of sites, connected by $J_{\perp}$, belonging to the two different rings).

In the two limiting regimes, the pair correlations can be computed also analytically. Interestingly, in each regime the intra-ring and the inter-ring pair correlations are equal. That is, the correlations between two sites are the same independently of the position of the two sites considered. In the superfluid regime, the ground state is condensed (3) and it yields $\eta_{\mathrm{BEC}}=(N-1) / N$. The pair correlation is independent of the number of sites since the atoms are completely delocalized. In the Mott insulator regime, the ground state has $\nu$ localized atoms in each site $(\nu \in \mathbb{Z})$ and from (4) it follows $\eta_{\mathrm{MI}}=1$. This means that if there is one atom in one site, the probability to find an atom in another site is one.

We have calculated numerically the pair correlations for two coupled trimers with $N=6$ (filling factor 1 ), as a function of $U / J_{\perp}$ for different values of $J / J_{\perp}$. Our numerical results are presented in Fig. 11. The top (bottom) panel corresponds to the intra-ring (inter-ring) pair

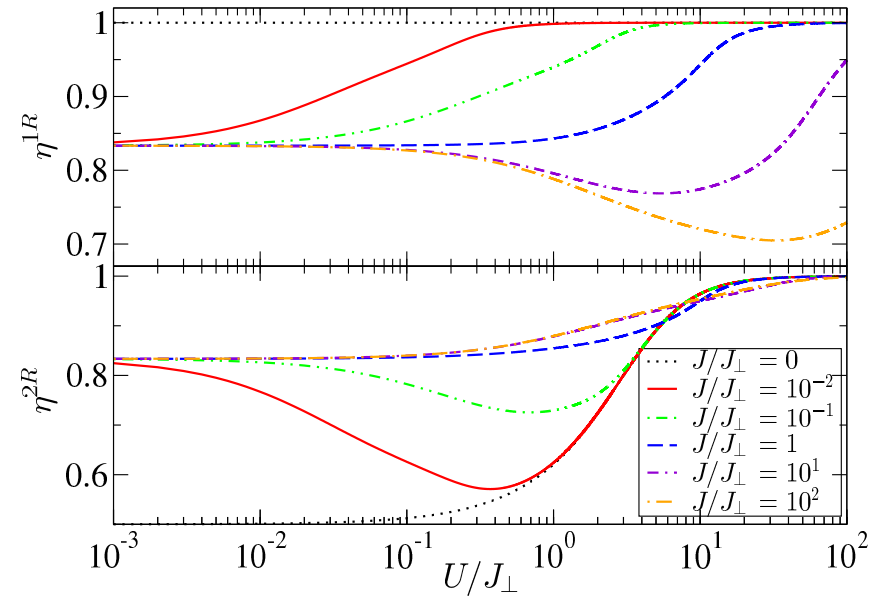

FIG. 11. Pair intra-ring correlation (top) and inter-ring correlation (bottom), as a function of $U / J_{\perp}$ for different values of $J / J_{\perp}$. In all cases, $N=6$ and $M=3$.

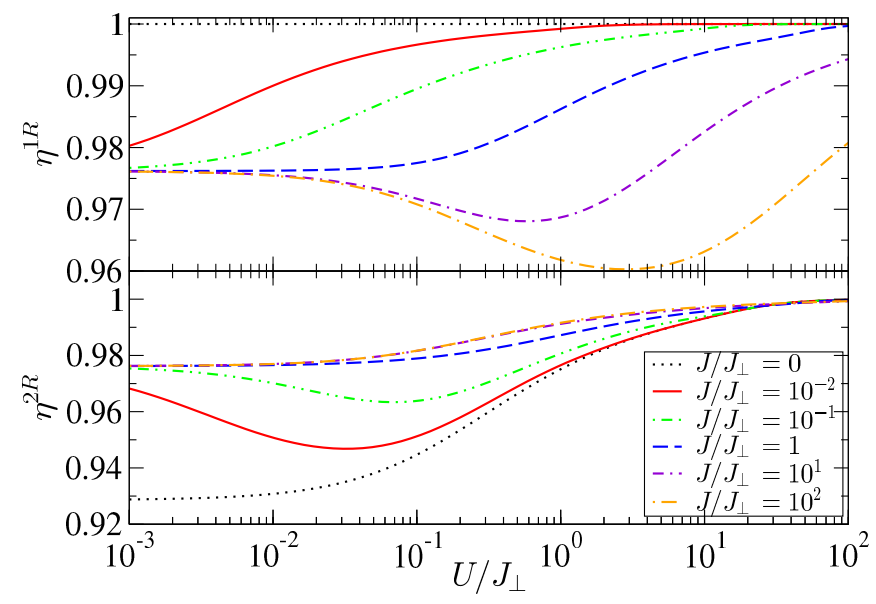

FIG. 12. Pair intra-ring correlation (top) and inter-ring correlation (bottom), as a function of $U / J_{\perp}$ for different values of $J / J_{\perp}$. In all cases, $N=42$ and $M=3$.

correlations, $\eta^{1 R}$ and $\eta^{2 R}$, respectively. As expected, for small values of the interaction the system is coherent and the intra-ring and inter-ring pair correlations tend both to the superfluid limit $(N-1) / N=5 / 6$, for all values of $J / J_{\perp}$ except for the particular case $J / J_{\perp}=0$.

In the top panel of Fig. 11 the pair intra-ring correlation between two adjacent sites of the same ring coupled by $J$ is depicted. A different behavior is appreciated when $J<J_{\perp}$ or $J>J_{\perp}$. When $J / J_{\perp}=0$ the two sites in the same ring are decoupled and the system behaves as three independent bosonic Josephson junctions. Since the two sites are independent, they become uncorrelated and $\eta^{1 R}=1$ for all values of $U / J_{\perp}$. In the other cases, when $J / J_{\perp} \neq 0$ and the interaction is small, the system is condensed and $\eta^{1 R} \rightarrow(N-1) / N$. 
As we have seen previously from the other magnitudes the system remains condensed for larger values of the interaction when $J$ increases, in particular when $J>J_{\perp}$. When $J / J_{\perp} \leq 1$ the intra-ring pair correlation has a smooth and monotonous behavior from the coherent limit $\eta_{\mathrm{BEC}}=(N-1) / N=5 / 6$ to the Mott insulator value $\eta_{\mathrm{MI}}=1$. Whereas for $J / J_{\perp}>1$ the system behaves as two weakly coupled rings and the competition between tunneling strengths causes the appearance of a minimum in the intra-ring pair correlation.

The inter-ring pair correlation $\eta^{2 R}$ is shown in the bottom panel of Fig. 11. Here we investigate the correlation between two sites that belong to different rings but are coupled by the tunneling strength $J_{\perp}$. The two limiting regimes appear clearly for all values $J / J_{\perp} \neq 0$ : the system remains coherent for small interactions $\left(\eta^{2 R} \rightarrow\right.$ $5 / 6$ ) and tends to fragmentation for large interactions $\left(\eta^{2 R} \rightarrow 1\right)$. In the particular case when $J / J_{\perp}=0$, the system acts as three independent double-wells, and the two sites behave as a bosonic Josephson junction, with $N=2$ particles and tunneling rate $J_{\perp}$, independently of the other sites; in this case when the interaction is small $\left(U / J_{\perp}<10^{-1}\right), \eta^{2 R}=1 / 2$, and increasing the interaction the system goes smoothly to the Mott insulator value $\eta_{\mathrm{MI}}=1$. The behavior of $\eta^{2 R}$ increases for all values of the tunneling ratio except when $J / J_{\perp}<1$, where a minimum appears for interactions $0.1<U / J_{\perp}<1$.

Notice that when $J / J_{\perp}=1$ the intra- and inter-ring couplings are the same, and there is no physical difference between two connected sites in the same ring or connected in different rings. This leads to the same curve with $J / J_{\perp}=1$ for $\eta^{1 R}$ and $\eta^{2 R}$ in both panels of Fig. 11 .

We show in Fig. 12 the intra-ring and inter-ring pair correlations for $N=42$ and $M=3$, as a function of $U / J_{\perp}$ for the same values of $J / J_{\perp}$ as in Fig. 11. The numerical results tend to the limiting values obtained analytically for commensurate systems in both limits, $U / J_{\perp} \ll 1$ and $U / J_{\perp} \gg 1$. When $J / J_{\perp} \neq 0$, and for large interactions (Mott insulator regime) $\eta^{1 R}=\eta^{2 R}=1$, and for small interactions $\eta^{1 R}=\eta^{2 R}=(N-1) / N=41 / 42$. Whereas when $J / J_{\perp}=0, \eta^{1 R}=1$ for all interactions, and $\eta^{2 R}=$ 1 for large interactions. In this latter tunneling ratio, the system behaves as three uncoupled double-wells with $N / 3=14$ atoms, therefore in the small interaction limit $\eta^{2 R}=(N / 3-1) /(N / 3)=13 / 14$ which is in agreement with the numerical results.

From Figs. 11 and 12 one can see that the general behavior of the pair correlations is similar for different commensurate number of atoms. However, the range of variation of $\eta^{1 R}$ and $\eta^{2 R}$ decreases when the number of trapped atoms increases, approaching to 1. Moreover, when $J / J_{\perp} \ll 1$ and the number of atoms increases, the system needs smaller values of $U / J_{\perp}$ to reach the noninteracting limit $\eta^{1 R}=\eta^{2 R}=(N-1) / N$ (see for instance the red curve $J / J_{\perp}=10^{-2}$ ).

The pair correlations in the double ring system change for large interactions in the Mott regime when we add (subtract) a particle from a commensurate situation. In- serting the corresponding incommensurate ground states (5) in the pair correlation definition, Eq. (11) it follows:

$$
\eta_{(i, j),(k, l)}^{ \pm}=4 M \frac{(M-1) \nu^{2}+\nu(\nu \pm 1)}{[(2 M-1) \nu+(\nu \pm 1)]^{2}},
$$

where \pm stands for adding (subtracting) an atom from the commensurate case $\nu \in \mathbb{Z}^{+}$, respectively. It is straightforward to see that the intra-ring and inter-ring correlations $\left(\eta^{1 R}\right.$ and $\left.\eta^{2 R}\right)$ are equal in this regime, since the extra particle (or vacancy) is equally populating all the sites. Eventually, one can verify that, both in the commensurate and in the incommensurate case, in the limit of large number of bosons $N$, the pair correlations tend to 1 for any value of $U / J_{\perp}$. This can be observed when comparing Figs. 11 and 12 and can be easily understood by computing the large- $N$ limit of $\eta_{\mathrm{BEC}}=(N-1) / N$ and of Eq. 12 .

\section{Reduced density matrix: Schmidt gap and entanglement entropy}

The spatial entanglement properties of the ground state $|\Psi\rangle$ can be characterized from the features of the reduced density matrix, obtained by performing a bipartite splitting of the system [67]. We consider the two rings as the natural partition in two subsystems in order to investigate the entanglement between them (see Fig. 15 for a sketch of the bipartite splitting of the system). From the density matrix $\rho=|\Psi\rangle\langle\Psi|$, tracing out the degrees of freedom of one ring, for example the bottom one, we obtain the reduced density matrix on the other subsystem, $\rho^{\uparrow}$, that describes the state of the top ring. The eigenvalues of $\rho^{\uparrow}$ are the Schmidt coefficients $\lambda_{k}^{\uparrow}$, which satisfy $\lambda_{1}^{\uparrow}>\lambda_{2}^{\uparrow}>\ldots$, and $\sum_{k} \lambda_{k}^{\uparrow}=1$. The coefficient $\lambda_{k}^{\uparrow}$ represents the probability of finding $k$ particles in the top ring without measuring the number of particles in the other ring [17.

The Schmidt gap is defined as the difference between the two largest coefficients of the Schmidt spectrum: $\Delta \lambda^{\uparrow}=\lambda_{1}^{\uparrow}-\lambda_{2}^{\uparrow}$, and it quantifies the entanglement of the two subsystems [67]. In the case of two subsystems without entanglement the Schmidt gap is $\Delta \lambda^{\uparrow}=1$, and the state of the system can be written as a product state. Whereas when $\Delta \lambda^{\uparrow}=0$ there is a large entanglement between the two subsystems and the total state of the system cannot be expressed as a product state.

An alternative description of the entanglement between the two rings (subsystems) can also be obtained from the single-ring von Neumann entropy, defined as $S^{\uparrow}=-\operatorname{Tr}\left(\rho^{\uparrow} \log \rho^{\uparrow}\right)$. Using the eigenvalues of the reduced density matrix, we can rewrite $S^{\uparrow}=$ $-\sum_{k} \lambda_{k}^{\uparrow} \log \lambda_{k}^{\uparrow}$. In the Mott insulator regime, since there is only one non-vanishing Schmidt coefficient $\lambda_{1}^{\uparrow}=1$, this leads to $S_{\mathrm{MI}}^{\uparrow}=0$. In the other limit, when the system is superfluid, the state is not separable in the two rings and 


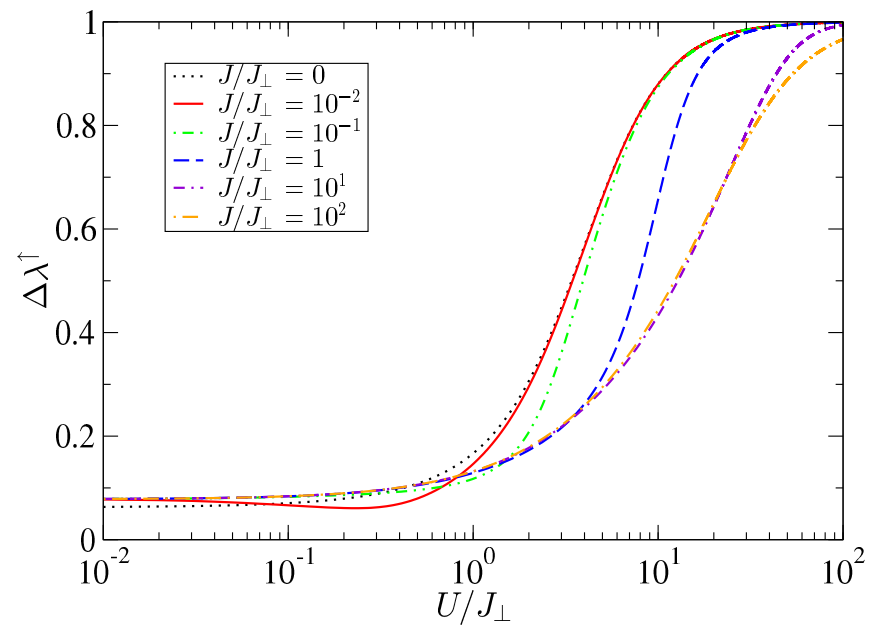

FIG. 13. Schmidt gap of the reduced density matrix of the top ring as a function of $U / J_{\perp}$ for different values of $J / J_{\perp}$. In all cases, $N=6$ and $M=3$.

the entanglement entropy is non zero, $S_{\mathrm{BEC}}^{\uparrow} \neq 0$. Tracing out one of the rings in the density matrix we obtain the following expression for the reduced density matrix $\rho^{\uparrow}$ (see equation $\mathrm{A} 5$ in the appendix),

$$
\rho^{\uparrow}=\sum_{\alpha, \beta} \sum_{m} \delta_{n(\alpha), n(\beta)} C_{\alpha, m} C_{\beta, m}^{*}|\alpha\rangle\langle\beta|,
$$

where $n(\alpha)$ is the number of particles of the Fock state $|\alpha\rangle$ in the top ring subspace, and $C_{\alpha, m}$ are the coefficients of the ground state in the Fock basis of the two rings system.

In Figs. 13 and 14 we plot the Schmidt gap and the entropy associated to the Schmidt coefficients of the subsystem formed by the top ring, with $N=6$ and $M=3$. The two limiting cases appear clearly in both figures: large tunneling couplings $U / J_{\perp} \ll 1$ (superfluid limit) and large interactions $U / J_{\perp} \gg 1$ (Mott insulator limit). The Mott insulator regime $\left(\Delta \lambda^{\uparrow}=1\right.$ and $\left.S^{\uparrow}=0\right)$ is reached for different values of the interaction strength $U / J_{\perp}$ depending on $J / J_{\perp}$. In this regime the state of the system is localized, with $\nu$ atoms in each site (when $\nu \in \mathbb{Z}$ ). Thus, it corresponds to only one Fock state that can be written as a product state of the top and bottom ring subspace. Therefore the two rings are decoupled. Since they are not entangled, it means that $\Delta \lambda^{\uparrow}=1$.

In the other limit, when $U / J_{\perp} \rightarrow 0$ and $U / J \rightarrow 0$ (with $\left.J / J_{\perp} \neq 0\right)$, we have checked that the first $(N+1)$ Schmidt coefficients that we have obtained numerically are equal to $\lambda_{k}^{\uparrow}=2^{-N}\left(\begin{array}{l}N \\ k\end{array}\right)$, with $k=0,1, \ldots, N$, that correspond to the Schmidt spectrum of a bosonic Josephson junction, see equation (A6) in the appendix, whereas the remaining Schmidt coefficients vanish. Thus, when the system approaches the coherent regime and $N$ is even ${ }^{1}$ the Schmidt

\footnotetext{
${ }^{1}$ Note that in the superfluid regime, due to the binomial distribution, the Schmidt gap will always vanish when $N$ is odd.
}

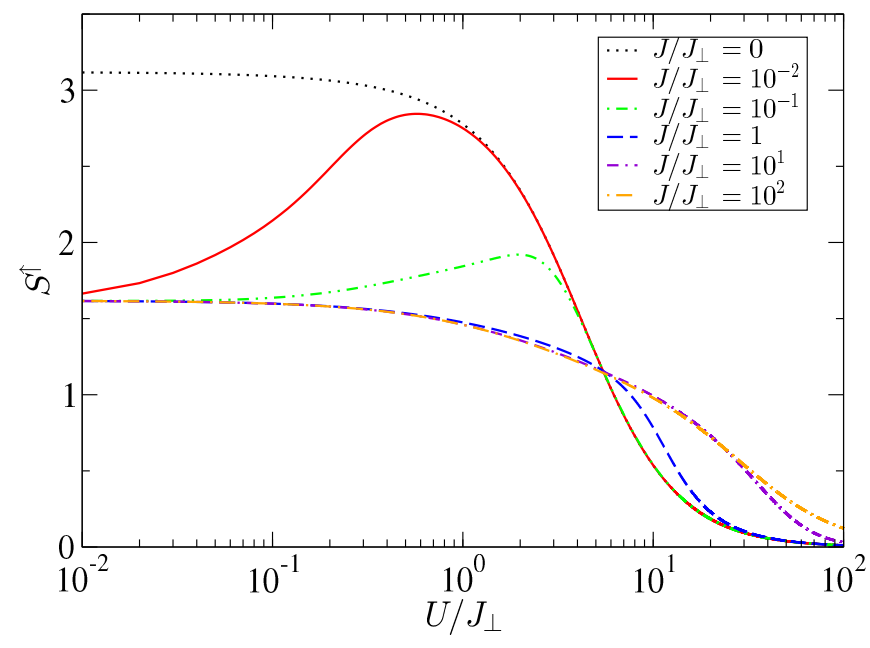

FIG. 14. Entanglement entropy $S^{\uparrow}$ as a function of $U / J_{\perp}$ for different values of $J / J_{\perp}$. In all cases, $N=6$ and $M=3$.

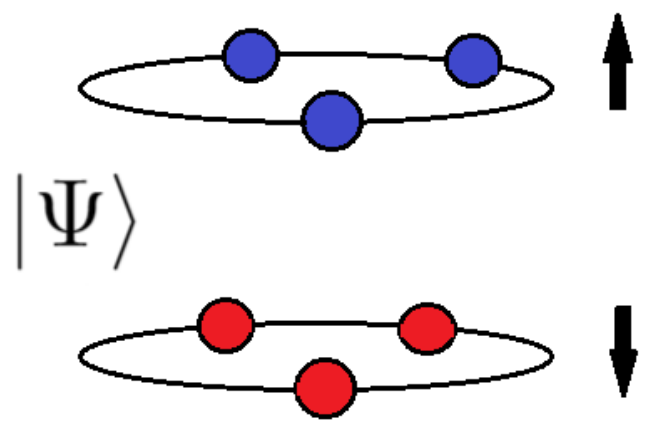

FIG. 15. Sketch of the bipartite splitting of the system. Each ring acts as a subsystem.

gap is given by $\Delta \lambda_{\mathrm{BEC}}^{\uparrow}=2^{-N}\left[\left(\begin{array}{c}N \\ N / 2\end{array}\right)-\left(\begin{array}{c}N \\ N / 2-1\end{array}\right)\right]$. In this regime the atoms are delocalized and therefore the probability to find a particle in one site is the same in all the sites, leading to a binomial distribution. In our case $\Delta \lambda^{\uparrow}=5 / 2^{6} \simeq 0.08$ as it appears in Fig. 13 when $U / J_{\perp} \ll 1$. In this regime, when one ring is traced out, the system presents the same features as one bosonic Josephson junction. Thus, each ring behaves as an effective single site, while the whole system acts as an effective bosonic Josephson junction between the two rings.

In Fig. 14 we show the entanglement entropy of the top ring. For large values of the interaction $U / J_{\perp}$ the system tends to the Mott insulator value $S^{\uparrow} \rightarrow 0$. On the contrary, in the coherent regime when $J / J_{\perp} \neq 0$, $S^{\uparrow} \simeq 1.62$, which corresponds to the entanglement entropy for one site in a bosonic Josephson junction (with $N=6$ ): $S^{\uparrow}=N \ln (2)-2^{-N} \sum_{k=0}^{N}\left(\begin{array}{c}N \\ k\end{array}\right) \ln \left(\begin{array}{c}N \\ k\end{array}\right)$ (see appendix). This limit is not reached when $J / J_{\perp}=0$ since in this case the tunneling between the sites in the same ring vanishes and the system corresponds to $M=3$ uncoupled bosonic Josephson junctions. In this situation 
the ground state is the product state of three individual Josephson wave functions which yields $S^{\uparrow} \simeq 3.1$.

From the above entanglement results, it follows that the ground state of two-coupled discrete circuits, when the number of bosons is even, can only be written as a product state of the two rings separately when the interaction is large in front of the tunneling (Mott insulator regime).

Let us consider a non commensurate number of atoms in the double ring. Figure 16 depicts the numerical calculations of the Schmidt gap (top panel) and the Schmidt entropy (bottom panel) of the top ring for a system with $M=3$ and 19 atoms. It corresponds to adding an extra atom from the commensurate case with $N=18$. In the limit of small interactions the system has the same physical behavior independently of the number of trapped atoms; there is a macroscopic occupation of a single particle state, and therefore $p_{1} \simeq 0$. The state of the system cannot be expressed as a product state of the two rings, then $\Delta \lambda^{\uparrow} \simeq 0$ and the two rings are entangled. Notice that for an odd number of particles, which is incommensurate with $2 M$ sites, the Schmidt gap always vanishes for any value of the interaction $U / J_{\perp}$, and therefore the system is always entangled, as it shown in the top panel of Fig. 16 .

As we have already previously discussed, in general, for large interactions the physical quantities are more sensitive to the non commensurability: the ground state is composed by a certain number of Fock states, due to the fact that the extra particle is shared between all the sites, and the two rings become entangled. In particular, when the system has one added (subtracted) atom from a commensurate case, the ground state can be described by Eq. (5). In this situation the reduced density matrix and therefore the Schmidt coefficients can be analytically computed. It follows that there are only two non-vanishing Schmidt coefficients $\lambda_{0}^{\uparrow}=\lambda_{1}^{\uparrow}=1 / 2$, which leads to $\Delta \lambda^{\uparrow}=0$ and $S^{\uparrow}=\ln (2)$. These limiting values are in agreement with the numerical results shown in Fig. 16 for large interactions $\left(U / J_{\perp}>10^{2}\right)$. As expected, this situation is clearly different from the commensurate one where $\Delta \lambda^{\uparrow}=1$ and $S^{\uparrow}=0$ (see Figs. 13 and 14 .

Eventually, it is worth emphasizing that the entanglement entropy has proved to be a rather effective indicator in detecting the occurrence of the superfluid-Mottinsulator transition (see Ref. [68, 69] and references therein). In general, if one considers the well-known $(J / U, \mu / U)$ phase diagram of the Bose-Hubbard model (where $\mu$ is the chemical potential), it is possible to draw constant-filling lines, both in the commensurate, and in the incommensurate case. Moving from the weakly interacting to the strongly interacting regime along a $\mathrm{com}$ mensurate constant-density line, the tip of the $\nu$-th Mott lobe is crossed before entering in the lobe itself. Accordingly, the entanglement entropy is known to exhibit a characteristic behavior [68]: it is non-zero in the superfluid region, maximum at the tip of the Mott lobe, and zero inside the latter. Indeed, this is exactly the scenario

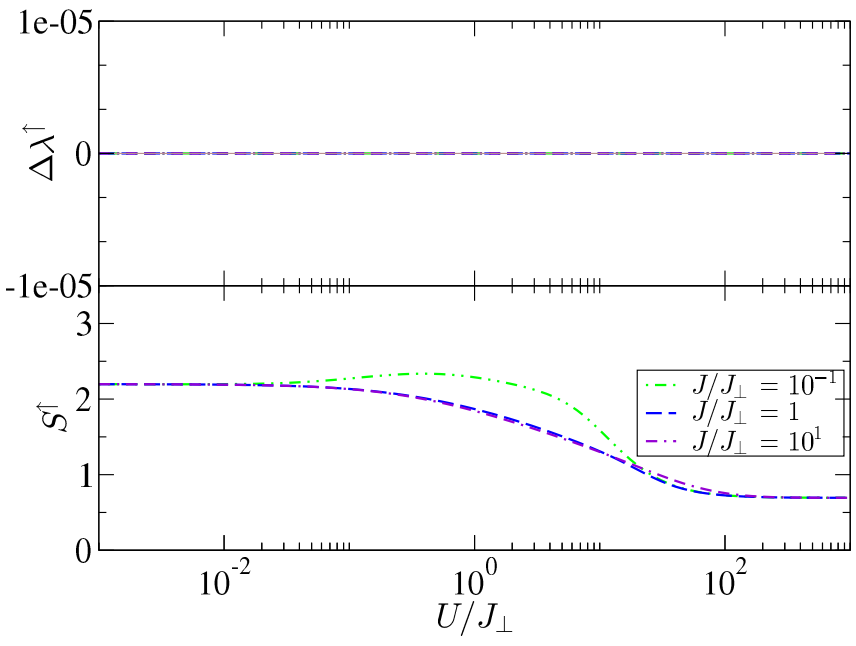

FIG. 16. Schmidt gap $\Delta \lambda^{\uparrow}$ (top panel) and entanglement entropy $S^{\uparrow}$ (bottom panel) of the top ring, as a function of $U / J_{\perp}$ for different values of $J / J_{\perp}$. In all cases, $N=19$ and $M=3$.

which emerges from the analysis of our two-ring system (see the red solid and the green dot-dashed lines in Fig. 14. Conversely, if one moves from the weakly interacting to the strongly interacting regime along an incommensurate constant-density line, no phase border is crossed. This is because such a line ends up being wedged between two adjacent Mott lobes in the $(J / U, \mu / U)$ plane, a circumstance corresponding to the fact that the superfluid solution is resilient to strong interactions. The entanglement entropy along this constant-density line is known to be a smooth function of the interaction and, as opposed to the commensurate case, it does not tend to zero in the strongly interacting regime. This is exactly what we observe when we compute the entanglement entropy of our two-ring system in the incommensurate case (see the bottom panel of Fig. 16.

\section{CONCLUSIONS}

In this work, we have considered a bosonic two-ring ladder, a system which is of particular interest in the emergent field of Atomtronics [5], as it can be suitably engineered to work as a supercurrent-based qubit device, it allows to explore magnetic-like phases in the presence of artificial gauge fields, and it is prone to disclose a rather rich dynamical scenario where persistent currents and vortices can tunnel between the rings. In addition, the two-ring ladder system does interpolate between other two classes of prototypical atomtronic devices: the famous double-well geometry (also known as bosonic Josephson junction) and the (single) BoseHubbard ring. Since we explored extended ranges of model parameters, the presented results have captured not only standard "asymptotic limits", but also interesting cross-over regimes, where the competition among 
different couplings is at its most crucial.

Within a simple analytical framework and by means of the exact numerical diagonalization of the system's Hamiltonian, we have investigated the static properties of the ground state. We have shown that the competition between the two tunneling strengths (intra-ring and inter-ring coupling), together with the interaction, determine the quantum properties of the two connected rings. We have also discussed the effects of the non commensurability when one atom is added (subtracted) from the commensurate situation, and we have shown that these effects become more important for large interactions.

First we have studied the single-particle ground state, which can be interpreted as different configurations of the same type of vortex states in both rings. Then we have considered the interacting system with different number of trapped atoms, providing a comprehensive analysis of the quantum features of the system. We have investigated the coherence and fragmentation properties, where we have found that the tunneling parameter $J$ is dominant in comparison with $J_{\perp}$. We have also studied the quantum correlations between sites. In general, the pair correlations increase with the interaction. However, there is a range of values, $J / J_{\perp}>1\left(J / J_{\perp}<1\right)$, where there is a competition between tunneling strengths that causes the appearance of a minimum in the intra-ring (interring) pair correlation for moderate values of the interaction. We have studied the entanglement of the ground state between the two rings computing its reduced density matrix. We have pointed out that in the superfluid case the non-null Schmidt coefficients of the system are the same as of a bosonic Josephson junction, and that the system is always entangled when the number of trapped atoms is incommensurate with the total number of sites of the system. Thus, the ground state can only be written as a product state in the Mott-insulator regime and with an integer value of the filling factor.

These results show a number of interesting quantum features such as correlations between two sites and quantum entanglement that can be used to design atomtronic circuits with two linearly coupled stacked rings.

\section{ACKNOWLEDGMENTS}

We thank useful discussions with Alessio Celi. A. E. also thanks Claudia Gonzalez, Jakub Janarek and Pere Mujal for interesting discussions. We acknowledge financial support from the Spanish MINECO and the Fondo Europeo de Desarrollo Regional (FEDER, EU) under Grants No. FIS2017-87801-P and FIS2017-87534$\mathrm{P}$, and from Generalitat de Catalunya under Grant No. 2017SGR533. A. E. is supported by Spanish MECD fellowship FPU15/03583.

\section{Appendix A: Reduced density matrix of one ring}

In order to obtain the set of eigenvalues $\left\{\lambda_{k}^{\uparrow}\right\}$ or Schmidt spectrum, one has to obtain the reduced density matrix $\rho^{\uparrow}$ by tracing out the degrees of freedom of the other ring. We present the general case of two coupled rings with $M$ sites each one, and $N$ particles. The dimension of the corresponding Hilbert space $\mathscr{H}$ is given by $\operatorname{dim}(\mathscr{H})=\mathcal{N}_{N}^{2 M}=$ $(N+2 M-1) ! /[N !(2 M-1) !]$. We consider the bipartite splitting in two subsystems which are given by the two rings $\mathscr{H}_{\uparrow}$ and $\mathscr{H}_{\downarrow}$. The dimension of this two subspaces is equal, given by $\operatorname{dim}\left(\mathscr{H}_{\uparrow}\right)=\sum_{n=0}^{N} \mathcal{N}_{n}^{M}=\frac{(N+1)}{M} \mathcal{N}_{N+1}^{M+N}$, but $\mathscr{H} \neq \mathscr{H}_{\uparrow} \otimes \mathscr{H}_{\downarrow}$. The many-body ground state expressed in the Fock basis of the whole system reads,

$$
|\Psi\rangle=\sum_{n_{1}, n_{2}, \ldots, n_{2 M}} C_{n_{1}, n_{2}, \ldots, n_{2 M}}\left|n_{1}, n_{2}, \ldots, n_{2 M}\right\rangle,
$$

where $n_{k}$ are number of particles on site $k$. The first sites $k=1, \ldots M$ correspond to the top ring, whereas $k=$ $M+1, \ldots 2 M$ to the bottom one. Since the total number of atoms is $N=\sum_{l=1}^{2 M} n_{l}$, we can rewrite the many-body state using this constraint:

$$
|\Psi\rangle=\sum_{\left\{n_{k}\right\}} C_{n_{1}, \ldots, n_{M}, n_{M+1}, \ldots, n_{2 M-1}}\left|n_{1}, \ldots, n_{M}, n_{M+1}, \ldots, n_{2 M-1}, N-\sum_{l=1}^{2 M-1} n_{l}\right\rangle .
$$

The eigenvectors of the Fock basis can be expressed as a product of the Fock basis elements corresponding to the subspaces of each ring:

$$
\left|n_{1}, \ldots, n_{M}, n_{M+1}, \ldots, n_{2 M}\right\rangle=\left|n_{1}, \ldots, n_{M}\right\rangle_{\uparrow} \otimes\left|n_{M+1}, \ldots, n_{2 M-1}, N-\sum_{l=1}^{2 M-1} n_{l}\right\rangle_{\downarrow}
$$


The reduced density matrix of the top ring, $\rho^{\uparrow}$, is obtained by tracing out the degrees of freedom of the other subsystem:

$$
\begin{aligned}
\rho^{\uparrow} & =\sum_{\left\{m_{k}\right\}}\left\langle m_{2 M}\left|\otimes \ldots \otimes\left\langle m_{M+1}|(|\Psi\rangle\langle\Psi|)| m_{M+1}\right\rangle \otimes \ldots \otimes\right| m_{2 M}\right\rangle \\
& =\sum_{\left\{m_{k}\right\}} \sum_{\left\{n_{k}\right\}} \sum_{\left\{n_{k}^{\prime}\right\}}\left(\prod_{l=M+1}^{2 M-1} \delta_{n_{l}, m_{l}} \delta_{n_{l}^{\prime}, m_{l}}\right) \delta_{N-\sum_{l=1}^{2 M-1} n_{l}, m_{2 M}} \delta_{N-\sum_{l=1}^{2 M-1} n_{l}^{\prime}, m_{2 M}} \\
& \times C_{n_{1}, \ldots, n_{2 M-1}} C_{n_{1}^{\prime}, \ldots, n_{2 M-1}^{\prime}}^{*}\left|n_{1}, \ldots, n_{M}\right\rangle\left\langle n_{1}^{\prime}, \ldots, n_{M}^{\prime}\right| \\
& =\sum_{\left\{m_{k}\right\}} \sum_{\left\{n_{k}\right\}} \sum_{\left\{n_{k}^{\prime}\right\}} \delta_{N-\sum_{l=1}^{M} n_{l}-\sum_{l=M+1}^{2 M-1}, m_{2 M}} \delta_{N-\sum_{l=1}^{M} n_{l}^{\prime}-\sum_{l=M+1}^{2 M-1}, m_{2 M}} \\
& \times C_{n_{1}, \ldots, n_{M}, m_{M+1}, \ldots, m_{2 M-1}} C_{n_{1}^{\prime}, \ldots, n_{M}^{\prime}, m_{M+1}, \ldots, m_{2 M-1}}\left|n_{1}, \ldots, n_{M}\right\rangle\left\langle n_{1}^{\prime}, \ldots, n_{M}^{\prime}\right| \\
= & \sum_{\left\{m_{k}\right\}} \sum_{\left\{n_{k}\right\}} \sum_{\left\{n_{k}^{\prime}\right\}} \delta_{\sum_{l=1}^{M} n_{l}, \sum_{l=1}^{M} n_{l}^{\prime}} C_{n_{1}, \ldots, n_{M}, m_{M+1}, \ldots, m_{2 M-1}} C_{n_{1}^{\prime}, \ldots, n_{M}^{\prime}, m_{M+1}, \ldots, m_{2 M-1}}^{*}\left|n_{1}, \ldots, n_{M}\right\rangle\left\langle n_{1}, \ldots, n_{M}\right| .
\end{aligned}
$$

Note that the condition $\delta_{\sum_{l=1}^{M} n_{l}, \sum_{l=1}^{M} n_{l}^{\prime}}$ in the previous expression is crucial, since only Fock states $\left|n_{1}, \ldots, n_{M}\right\rangle$ and $\left\langle n_{1}^{\prime}, \ldots, n_{M}^{\prime}\right|$ that have the same number of particles, i.e, $\sum_{l=1}^{M} n_{l}=\sum_{l=1}^{M} n_{l}^{\prime}$, will contribute. This will be very useful in order to perform numerical computations.

The following steep is to diagonalize $\rho^{\uparrow}$ in order to obtain the eigenvalues $\left\{\lambda_{i}^{\uparrow}\right\}$ and from the two largest ones compute the Schmidt gap. In general, for any number of sites $M$ and particles $N$ the matrix $\rho^{\uparrow}$ is given by:

$$
\rho^{\uparrow}=\sum_{\alpha, \beta}^{\operatorname{dim}\left(\mathscr{H}_{\uparrow}\right)} \sum_{m} \delta_{n(\alpha), n(\beta)} C_{\alpha, m} C_{\beta, m}^{*}|\alpha\rangle\langle\beta|,
$$

where $n(\alpha)$ is the number of particles of the Fock state $|\alpha\rangle$ in the subspace of the top ring, $\mathscr{H}_{\uparrow}$.

This expression is valid for any system in which the bipartite splitting leads to two equal subsystems with the same dimension. For instance, consider $N$ bosons in a Josephson junction with two sites. The many-body state in the superfluid regime (when the tunneling is larger than the interaction) is given by:

$$
\left|\Psi_{\mathrm{sf}}\right\rangle=\frac{1}{2^{N / 2}} \sum_{n=0}^{N} \sqrt{\left(\begin{array}{c}
N \\
n
\end{array}\right)}|n, N-n\rangle .
$$

The bipartite splitting corresponds to each site. Therefore, the eigenstates of the subspace $\mathscr{H}_{\uparrow}$ are the Fock states of the top site like $|n\rangle$. The reduced density matrix $\rho^{\uparrow}$ is diagonal, whose eigenvalues (and the Schmidt spectrum) are given by the binomial distribution $\lambda_{k}=2^{-N}\left(\begin{array}{l}N \\ n\end{array}\right)$. This leads to the following analytical expressions for the Schmidt gap $\Delta \lambda^{\uparrow}=2^{-N}\left[\left(\begin{array}{c}N \\ N / 2\end{array}\right)-\left(\begin{array}{c}N \\ N / 2-1\end{array}\right)\right]$ (for $N$ even), and the entanglement entropy $S_{\uparrow}=N \ln (2)-2^{-N} \sum_{k=0}^{N}\left(\begin{array}{c}N \\ k\end{array}\right) \ln \left(\begin{array}{c}N \\ k\end{array}\right)$.

[1] L. Amico, G. Birkl, M. Boshier, and L.-C. Kwek, New J. Phys. 19, 020201 (2017)

[2] B. T. Seaman, M. Krämer, D. Z. Anderson, and M. J. Holland, Phys. Rev. A 75, 023615 (2007)

[3] S. Safaei, L.-C. Kwek, R. Dumke, and L. Amico, Phys. Rev. A 100, 013621 (2019).

[4] S. Safaei, B. Grémaud, R. Dumke, L.-C. Kwek, L. Amico, and C. Miniatura, Phys. Rev. A 97, 042306 (2018).

[5] L. Amico, M. Boshier, G. Birkl, A. Minguzzi, C. Miniatura, L. C. Kwek, D. Aghamalyan, V. Ahufinger, N. Andrei, A. S. Arnold, M. Baker, T. A. Bell, T. Bland, J. P. Brantut, D. Cassettari, F. Chevy, R. Citro, S. D. Palo, R. Dumke, M. Edwards, R. Folman, J. Fortagh,
S. A. Gardiner, B. M. Garraway, G. Gauthier, A. Günther, T. Haug, C. Hufnagel, M. Keil, W. von Klitzing, P. Ireland, M. Lebrat, W. Li, L. Longchambon, J. Mompart, O. Morsch, P. Naldesi, T. W. Neely, M. Olshanii, E. Orignac, S. Pandey, A. Pérez-Obiol, H. Perrin, L. Piroli, J. Polo, A. L. Pritchard, N. P. Proukakis, C. Rylands, H. Rubinsztein-Dunlop, F. Scazza, S. Stringari, F. Tosto, A. Trombettoni, N. Victorin, D. Wilkowski, K. Xhani, and A. Yakimenko, "Roadmap on atomtronics," (2020), arXiv:2008.04439 [cond-mat.quant-gas].

[6] T. Haug, R. Dumke, L.-C. Kwek, and L. Amico, Quantum Sci. Technol. 4, 045001 (2019).

[7] I. Chiorescu, Y. Nakamura, C. J. P. M. Harmans, and 
J. E. Mooij, Science 299, 1869 (2003)

[8] I. M. Georgescu, S. Ashhab, and F. Nori, Rev. Mod. Phys. 86, 153 (2014)

[9] A. C. Mathey and L. Mathey, New J. Phys. 18, 055016 (2016)

[10] T. Haug, J. Tan, M. Theng, R. Dumke, L.-C. Kwek, and L. Amico, Phys. Rev. A 97, 013633 (2018)

[11] Y. Wu and X. Yang, J. Opt. Soc. Am. B 23, 1888 (2006)

[12] L. Morales-Molina, S. A. Reyes, and M. Orszag, Phys. Rev. A 86, 033629 (2012).

[13] P. Buonsante, P. Kevrekidis, V. Penna, and A. Vezzani, J. Phys. B: At. Mol. Opt. Phys. 39, S77 (2006).

[14] A. Gallemí, M. Guilleumas, R. Mayol, and A. Sanpera, Phys. Rev. A 88, 063645 (2013).

[15] L. Dell'Anna, G. Mazzarella, V. Penna, and L. Salasnich, Phys. Rev. A 87, 053620 (2013).

[16] A. Gallemí, M. Guilleumas, J. Martorell, R. Mayol, A. Polls, and B. Juliá-Díaz, New J. Phys. 17, 073014 (2015)

[17] A. Gallemí, M. Guilleumas, J. Martorell, R. Mayol, A. Polls, and B. Juliá-Díaz, New J. Phys. 18, 075005 (2016)

[18 D. W. Hallwood, J. Burnett, and J. Dunningham, New J. Phys. 8, 180 (2006).

[19] C. Lee, T. J. Alexander, and Y. S. Kivshar, Phys. Rev. Lett. 97, 180408 (2006).

[20] G. Arwas, A. Vardi, and D. Cohen, Phys. Rev. A 89, 013601 (2014).

[21] G. S. Paraoanu, Phys. Rev. A 67, 023607 (2003).

[22] A. Muñoz Mateo, V. Delgado, M. Guilleumas, R. Mayol, and J. Brand, Phys. Rev. A 99, 023630 (2019).

[23] D. Aghamalyan, L. Amico, and L. C. Kwek, Phys. Rev. A 88, 063627 (2013)

[24] L. Amico, D. Aghamalyan, F. Auksztol, H. Crepaz, R. Dumke, and L. C. Kwek, Scientific reports 4, 4298 (2014).

[25] J. Mooij, T. Orlando, L. Levitov, L. Tian, C. H. Van der Wal, and S. Lloyd, Science 285, 1036 (1999).

[26] M. Di Dio, S. De Palo, E. Orignac, R. Citro, and M.-L. Chiofalo, Phys. Rev. B 92, 060506 (2015).

[27] E. Orignac and T. Giamarchi, Phys. Rev. B 64, 144515 (2001).

[28] E. Orignac, R. Citro, M. Di Dio, S. De Palo, and M.-L. Chiofalo, New Journal of Physics 18, 055017 (2016).

[29] E. Orignac, R. Citro, M. Di Dio, and S. De Palo, Phys. Rev. B 96, 014518 (2017).

[30] R. Citro, S. De Palo, M. Di Dio, and E. Orignac, Phys. Rev. B 97, 174523 (2018).

[31] M. Filippone, C.-E. Bardyn, and T. Giamarchi, Phys. Rev. B 97, 201408 (2018).

[32] T. Haug, L. Amico, R. Dumke, and L.-C. Kwek, Quantum Sci. Technol. 3, 035006 (2018).

[33] N. Victorin, F. Hekking, and A. Minguzzi, Phys. Rev. A 98, 053626 (2018).

[34] N. Victorin, T. Haug, L.-C. Kwek, L. Amico, and A. Minguzzi, Phys. Rev. A 99, 033616 (2019).

[35] M. Piraud, F. Heidrich-Meisner, I. P. McCulloch, S. Greschner, T. Vekua, and U. Schollwöck, Phys. Rev. B 91, 140406 (2015)

[36] A. Oliinyk, I. Yatsuta, B. Malomed, and A. Yakimenko, Symmetry 11, 1312 (2019).

[37] A. Oliinyk, A. Yakimenko, and B. Malomed, Journal of Physics B: Atomic, Molecular and Optical Physics 52, 225301 (2019).
[38] A. Oliinyk, B. Malomed, and A. Yakimenko, Communications in Nonlinear Science and Numerical Simulation 83, 105113 (2020).

[39] A. Richaud and V. Penna, Phys. Rev. A 96, 013620 (2017)

[40] I. Lesanovsky and W. von Klitzing, Phys. Rev. Lett. 98, $050401(2007)$

[41] E. Nicolau, J. Mompart, B. Juliá-Díaz, and V. Ahufinger, Phys. Rev. A 102, 023331 (2020).

[42] A. Escrivà, A. Muñoz Mateo, M. Guilleumas, and B. Juliá-Díaz, Phys. Rev. A 100, 063621 (2019).

[43] A. Gallemí, A. Muñoz Mateo, R. Mayol, and M. Guilleumas, New J. Phys. 18, 015003 (2016).

[44] D. Aghamalyan, N. T. Nguyen, F. Auksztol, K. S. Gan, M. M. Valado, P. C. Condylis, L.-C. Kwek, R. Dumke, and L. Amico, New J. Phys. 18, 075013 (2016)

[45] M. Albiez, R. Gati, J. Fölling, S. Hunsmann, M. Cristiani, and M. K. Oberthaler, Phys. Rev. Lett. 95, 010402 (2005).

[46] T. Schumm, S. Hofferberth, L. M. Andersson, S. Wildermuth, S. Groth, I. Bar-Joseph, J. Schmiedmayer, and P. Krüger, Nature physics 1, 57 (2005).

[47] G. Spagnolli, G. Semeghini, L. Masi, G. Ferioli, A. Trenkwalder, S. Coop, M. Landini, L. Pezzè, G. Modugno, M. Inguscio, A. Smerzi, and M. Fattori, Phys. Rev. Lett. 118, 230403 (2017).

[48] G. Valtolina, A. Burchianti, A. Amico, E. Neri, K. Xhani, J. A. Seman, A. Trombettoni, A. Smerzi, M. Zaccanti, M. Inguscio, et al., Science 350, 1505 (2015).

[49] A. Burchianti, F. Scazza, A. Amico, G. Valtolina, J. Seman, C. Fort, M. Zaccanti, M. Inguscio, and G. Roati, Physical review letters 120, 025302 (2018).

[50] R. Islam, R. Ma, P. M. Preiss, M. Eric Tai, A. Lukin, M. Rispoli, and M. Greiner, Nature 528, 77 (2015).

[51] M. Cheneau, P. Barmettler, D. Poletti, M. Endres, P. Schausz, T. Fukuhara, C. Gross, I. Bloch, C. Kollath, and S. Kuhr, Nature 481, 484 (2012).

[52] M. R. Sturm, M. Schlosser, R. Walser, and G. Birkl, Phys. Rev. A 95, 063625 (2017).

[53] S. Raghavan, A. Smerzi, S. Fantoni, and S. R. Shenoy, Phys. Rev. A 59, 620 (1999).

[54] A. Keles and M. O. Oktel, Phys. Rev. A 91, 013629 (2015)

[55] J. M. Zhang and R. X. Dong, Eur. J. Phys. 31, 591 (2010)

[56] D. Raventós, T. Graß, M. Lewenstein, and B. Juliá-Díaz, J. Phys. B: At. Mol. Opt. Phys. 50, 1361 (2017).

[57] L. Amico, A. Osterloh, and F. Cataliotti, Phys. Rev. Lett. 95, 063201 (2005)

[58] M. Atala, M. Aidelsburger, M. Lohse, M. T. Barreiro, B. Paredes, and I. Bloch, Nature Physics 10, 237208 (2014).

[59] E. J. Mueller, T.-L. Ho, M. Ueda, and G. Baym, Phys. Rev. A 74, 033612 (2006).

[60] R. A. Pepino, J. Cooper, D. Z. Anderson, and M. J. Holland, Phys. Rev. Lett. 103, 140405 (2009).

[61] I. Danshita and A. Polkovnikov, Phys. Rev. A 84, 063637 (2011).

[62] F. Lingua, A. Richaud, and V. Penna, Entropy 20, 84 (2018).

[63] V. Penna and A. Richaud, J. Phys. Conf. Ser. 1206, 10242 (2019).

[64] A. Richaud and V. Penna, New Journal of Physics 20, 105008 (2018). 
[65] A. Richaud and V. Penna, Phys. Rev. A 100, 013609 (2019).

[66] A. Richaud, A. Zenesini, and V. Penna, Sci. Rep. 9, 6908 (2019).

[67] G. De Chiara and A. Sanpera, Rep. Prog. Phys. 81, 074002 (2018).
[68] I. Frérot and T. Roscilde, Phys. Rev. Lett. 116, 190401 (2016).

[69] I. Morera, I. Frérot, A. Polls, and B. Juliá-Díaz, Phys. Rev. Research 2, 033016 (2020). 